\title{
Taxonomy and Natural History of the Crematogaster (Decacrema)-group (Hymenoptera: Formicidae) in Madagascar
}

\author{
BONNIE B. BLAIMER \\ Department of Entomology, University of California-Davis, One Shields Ave, Davis, CA 95616. E-mail: bbblaimer@ucdavis.edu
}

\section{Table of content}

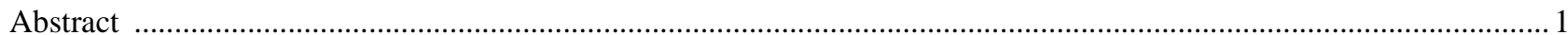

Introduction

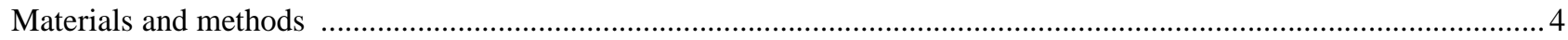

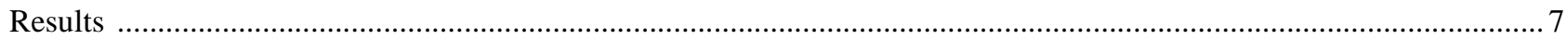

Key to the Subgenera of the Crematogaster of the Malagasy region: Workers ........................................................... 7

Key to the Subgenera of the Crematogaster of the Malagasy region: Queens (as far as queens are known) .................. 7

List of Crematogaster species/subspecies currently classified under Oxygyne, Orthocrema, Mesocrema and Cremato-

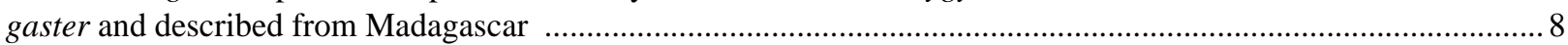

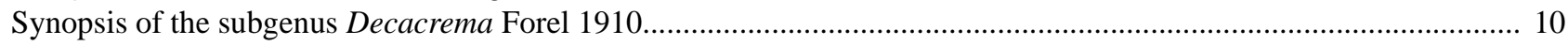

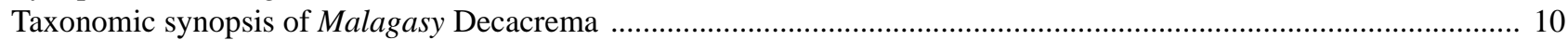

Key to the workers of the Crematogaster (Decacrema)-group, Malagasy region ................................................ 14

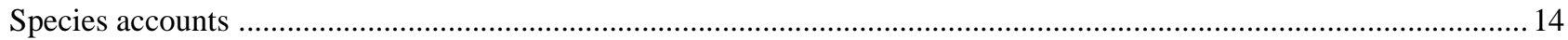

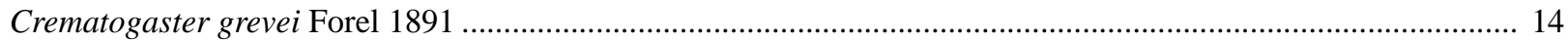

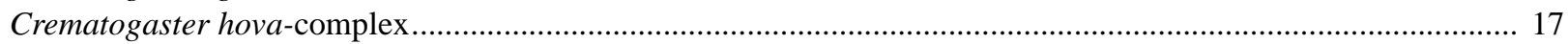

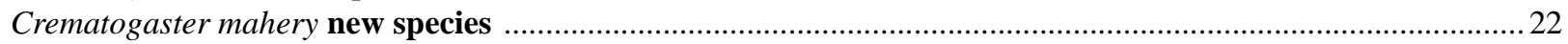

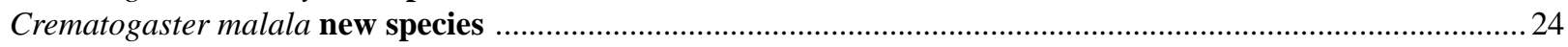

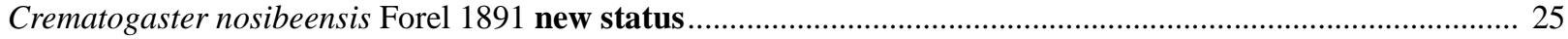

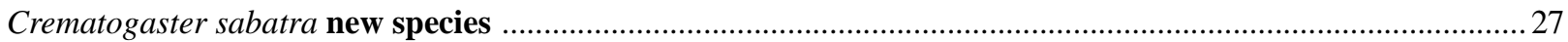

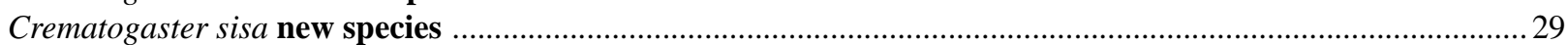

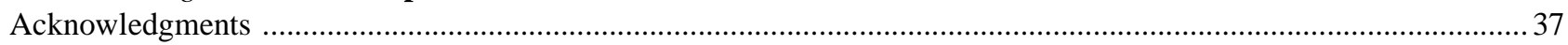

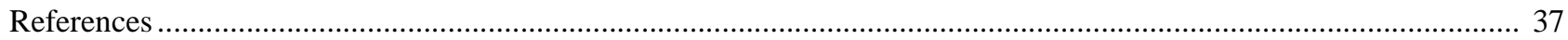

\begin{abstract}
The taxonomy of the Malagasy Crematogaster (Decacrema) is revised and a total of six distinct species and one speciescomplex are recognized. Malagasy Decacrema are widespread ants in Madagascar that nest almost exclusively arboreally, either in carton nests or inside dead twigs or branches, and often tend Coccoidea. Four species are newly described here: Crematogaster mahery sp. nov., C. malala sp. nov., C. sabatra sp. nov. and $C$. sisa sp. nov.. The species status of Crematogaster grevei Forel 1891 is confirmed by the study, and one described subspecies, C. hova nosibeensis Forel 1891 is raised to species level (C. nosibeensis stat. nov.) and a neotype designated. Crematogaster hova latinoda Forel 1891 is synonymised under $C$. hova Forel 1887, and the taxonomic status of C. hova, C. ensifera Forel 1910 and $C$. schencki Forel 1891 is further investigated, but could not be resolved with methods employed here. The $C$. hovacomplex, a species-complex consisting of five morphological forms, is hence defined and the three described species above are associated with these morphotypes. Full descriptions including natural history information are presented for all species and the species-complex, as well as images, distribution maps and a species-identification key to the workers of Malagasy Decacrema. A diagnosis of the Malagasy Decacrema and identification keys to the subgenera of the ant genus Crematogaster in the Malagasy region are also provided.
\end{abstract}

Key words: Myrmicinae, arboreal ant, species-complex, subgenera, carton nest 


\section{INTRODUCTION}

The genus Crematogaster Lund (1831) is a widespread, globally distributed group within the largest subfamily of ants, the Myrmicinae (Brown, 1973; Hölldobler \& Wilson, 1990; Longino, 2003; Ward, 2010) and currently comprises more than 780 valid species and subspecies names (Bolton et al., 2007). Crematogaster ants are widespread, but reach their highest diversity and abundance in tropical and subtropical regions. These ants are generally found in forest, woodland and shrubby habitats, where they form a conspicuous and often dominant element of the fauna. Most tropical Crematogaster species nest arboreally, but some tropical and many temperate zone species nest in the ground (e.g. Hosoishi et al., 2010). Worker ants of this genus are easily recognized by a few unique morphological features, including the dorsal attachment of the postpetiole to the $4^{\text {th }}$ abdominal segment and the absence of a dorsal petiolar node, which give the ants the ability of flexing the gaster forwards over the mesosoma while the petiole is pressed tightly against the propodeum (Buren, 1959). This is an aggressive response to every invader, enabling the ants to use their remarkable spatulate sting for the topical application of their venom - which apparently is efficient in repelling, if not killing other ant opponents (Marlier et al., 2004). Because of this distinctive morphology the monophyly of Crematogaster has never been doubted, although phylogenetic work on the genus is to date largely lacking and the closest myrmicine relative to Crematogaster remains uncertain. Bolton (2003) suggested the enigmatic Asian-endemic genus Recurvidris Bolton and placed it as sole member aside from Crematogaster in the tribe Crematogastrini, but preliminary molecular phylogenetic work does not support the hypothesis that Crematogaster and Recurvidris are sister taxa (P. S. Ward, pers. comm.).

Contrasting with the unquestioned monophyly of the genus Crematogaster is a state of taxonomic and nomenclatural chaos at the species level, generated by the considerable morphological variability within some species that is paired often with close similarity between species. Earlier taxonomists (e.g. Arnold, 1920; Brown, 1973) have suggested that the genus is burdened by synonymy, and more recent work confirms the persistence of this nomenclatural disarray (Longino, 2003; Ward, 2007, 2010). Species of Crematogaster have further been grouped into several 'subgenera' on the basis of morphological features. A key to 12 'subgenera' was presented by Santschi (1918), but additions and regroupings were made by various authors (Emery, 1922; Mann, 1919; Santschi, 1928; Wheeler, 1927, 1936). In consequence, Bolton et al. (2007) currently list 16 'subgenera' for Crematogaster, although two of them are monotypic. The monophyly of most of these groupings is highly doubtful - they should therefore be treated merely as hypotheses for species-groups and must be closely scrutinized. They can however still provide a useful means to break down any alphataxonomic work on this species-rich genus into manageable subunits. Recent species-level taxonomic work on Crematogaster has been accomplished either on a regional scale (Longino, 2003) or a subgeneric basis (Hosoishi \& Ogata, 2009), and the approach presented here is a hybrid of the two in the form of a regional subgeneric revision.

Madagascar and the Malagasy Crematogaster. Madagascar often has been dubbed the "eighth continent" because of its unique biota characterized by high species diversity and endemism (Goodman \& Benstead, 2005), and it is counted among the world's most prominent biodiversity hotspots (Myers et al., 2000; Yoder \& Nowak, 2006). The island's vegetation can be broadly divided into five major biomes: the Eastern Region, the Sambirano Region, the Central Region, the Western Region and the Southern Region (Gautier \& Goodman, 2003). The latter two are arid regions whose dry deciduous and spiny forests harbour a distinctly different faunal assemblage from the humid and sub-humid forests of the Eastern, Sambirano and Central Regions. Knowledge on distributions and species-level taxonomy are critical data for ongoing efforts in designating conservation priorities in Madagascar (Kremen et al., 2008), and recent extensive and methodical surveys of the arthropod and especially the ant fauna of Madagascar (Fisher, 2005; Fisher \& Penny, 2008) now present a solid basis for more advanced alpha-taxonomic work.

A total of 20 species and 11 subspecies of Crematogaster have been previously described from Madagascar (Bolton et al., 2007), currently classified in five 'subgenera': Decacrema Forel (1910), Oxygyne Forel (1901), Orthocrema Santschi (1918), Mesocrema Santschi (1928) and Crematogaster sensu stricto . In Madagascar, ants of the genus Crematogaster occur in all forest habitats and probably are the most dominant 
and abundant ants in the canopy, rivalled only by Camponotus, Tetraponera and Pheidole. Weaver ants (Oecophylla), which dominate arboreal habitats throughout the rest of the Palaeotropics, are absent from Madagascar; this could have presented ecological and evolutionary opportunities for other genera such as Crematogaster and led to increased species diversification (Fisher, 2003). In Madagascar, nest constructions such as carton nests are built exclusively by Crematogaster species from at least three of the 'subgenera' (Decacrema, Oxygyne and Crematogaster). Carton nests are made from masticated plant material that the ants then plaster together in layers (see Weissflog, 2001). Other preferred arboreal nesting habitats are dead twigs or branches in the canopy or lower vegetation, and beneath canopy moss and epiphyte mats; a few species stray from this largely arboreal lifestyle and nest on the ground, either in rotten logs or under stones. Most arboreal species seem to also forage extensively on the ground, but this aspect of life history needs further investigation. In contrast to many other tropical regions, Crematogaster is not known to be involved in any close associations with plants in Madagascar (Fisher, pers. comm.).

The subgenus Decacrema Forel. A specialised 'plant-ant' habit has brought the Decacrema-group the most attention of all 'subgenera' within Crematogaster. Species from South-East Asia assigned to this 'subgenus' have mutualistic associations with Macaranga trees, and these relationships have been investigated as model systems for co-evolution (e.g. Feldhaar et al., 2003; Quek et al., 2004). The Crematogaster (Decacrema)-group has been morphologically defined by having 10-segmented antennae versus 11 segments (in workers and queens, males have 12 segments) in all other 'subgenera' of Crematogaster. Species from Madagascar, Sub-Saharan Africa, Singapore and the Malay Archipelago have been assigned to Decacrema (Emery, 1922; Hosoishi \& Ogata, 2010), for a total of 24 species and subspecies names (Bolton et al., 2007). Although the ecology of the Macaranga-inhabiting Decacrema has been well circumscribed (Feldhaar et al., 2010; Fiala et al., 1999) and their taxonomy is undergoing revision (Feldhaar et al, in prep.), taxonomic and biological information on the remaining Asian, African or Malagasy species in the group is very sparse. The cohesion of Decacrema as a group however has been challenged by preliminary data and there is compelling molecular evidence that at least the Macaranga-Decacrema and the Malagasy Decacrema do not form a clade (Blaimer, unpubl.). The morphology of all but one examined African species further refutes a close relationship to the Decacrema of Madagascar (Blaimer, pers. observ.) and warrants further study.

Natural history and taxonomy of Decacrema in Madagascar. Molecular and morphological data strongly support the monophyly of the Malagasy Decacrema (Blaimer, unpubl.) and thus justify their treatment as a cohesive natural group in this revision. In Madagascar, Decacrema ants are found throughout all forest habitats within the five biomes on the island. Nesting habits are arboreal except for one species that is known to occasionally nest on the ground and is adapted to drier habitats. Many of the canopy-nesting species construct carton nests around small branches or attached to tree trunks, or alternatively nest in dead branches or twigs. It is unclear whether these nesting habits are species-specific. Malagasy Decacrema are medium to large-sized ants compared to other Crematogaster species in this region, and they often seem to be the dominant element of the Crematogaster, or even of the entire canopy ant fauna where they occur. Partly this may be mediated by the carton-nesting ability that allows for large and often polydomous colonies. Carton nesters often tend and house mealybugs in the main nest or in special carton shelters, and both twigand carton-nesting species can further be found living in association with myrmecophilous beetles (Blaimer, pers. observ.).

Four described species and two subspecies currently represent Decacrema in Madagascar: Crematogaster grevei Forel 1891, C. hova Forel 1887, C. hova nosibeensis Forel 1891, C. hova latinoda Forel 1892, C. ensifera Forel 1910 and $C$. schencki Forel 1891 (Emery, 1922). The taxonomy of these species has not been revised since their descriptions. Poor geographic sampling and a confusing morphological variability hampered correct taxonomic inference at that time. However, even with the aid of extensive sampling from biodiversity surveys in Madagascar and additional lines of evidence from molecular data (see below), it is not possible to resolve all species boundaries within the Malagasy Decacrema clade. The following revisionary work hence recognizes a total of six species and delineates a species-complex with three of the described species names (C. hova, $C$. ensifera and $C$. schencki) associated. Defining the problem here is only the initial 
step to its solution, and this question will need to be approached with other methods to achieve full resolution. This study also aims to be a first descriptive work on the natural history of the Malagasy Decacrema and is intended to spur further ecological studies on this highly successful, intriguing group of arboreal ants in Madagascar.

\section{MATERIALS AND METHODS}

Specimens were examined and/or deposited in the following collections:

CASC California Academy of Sciences, San Francisco, CA, USA

BBBC B.B. Blaimer Collection, University of California at Davis, CA, USA

MHNG Muséum d'Histoire Naturelle, Genève, Switzerland

NHMB Naturhistorisches Museum, Basel, Switzerland

PSWC P. S. Ward Collection, University of California at Davis, CA, USA

SAMC South African Museum, Cape Town, South Africa

UCDC Bohart Museum of Entomology, University of California, Davis, USA

ZMBH Museum für Naturkunde der Humboldt Universität, Berlin, Germany

ZSM Zoologische Staatssammlung München, Munich, Germany

All morphological observations were made with a Leica MZ12.5 stereomicroscope. Standard measurements (in $\mathrm{mm}$ ) were taken at 50x, or rarely at 25x (some queen measurements) with a Wild M5A stereomicroscope. and a dual-axis Nikon micrometer wired to a digital readout. Measurements were recorded to the nearest $0.001 \mathrm{~mm}$, but for brevity are presented here to the second decimal place. Ranges are always presented as minimum - maximum values. A minimum of 10 workers were measured for each species, where possible representing the entire distribution range. As far as queens could be obtained from colony collections in association with workers, the same measurements as for workers were taken, as well as additional queenspecific measurements. The abbreviations used for measurements and indices are given below. Standardization of measurements follows Longino (2003) where applicable, except as follows. Procedure for LHT follows Ward (2001), and LBI, MSNW, MSNL and MSNI are defined below for the purpose of this study. Indices are presented as decimal fractions to two decimal places (rather than percents as in Longino, 2003).

\section{Measurements and indices}

HW $\quad$ Maximum head width including eyes, in full face view (Fig. 1).

HL Head length; perpendicular distance from line tangent to rearmost points of vertex margin to line tangent to anterior most projections of clypeus, in full face view (Fig. 1).

EL $\quad$ Eye length, measured along maximum diameter (Fig. 1).

SL Scape length, length of scape shaft from apex to basal flange, not including basal condyle and neck. If scape is strongly arched, this measurement is taken as perpendicular distance between line tangent to apex and line tangent to basal flange (Fig. 2).

PTL Petiole length; measured in lateral profile as the distance from dorso-posterior margin of segment to anterior inflection point where petiole curves up to condyle (Fig. 5) [note that this interpretation slightly differs from Longino (2003)].

PTH Petiole height; measured in lateral profile as vertical distance from ventral margin to highest point of dorso-posterior margin (Fig. 5).

PTW Petiole width; maximum width of petiole in dorsal view (Fig. 6).

PPL Postpetiole length; measured in dorsal view at an angle that maximizes length

(Fig. 6). 
(Fig. 6).

WL Weber's length; measured in lateral profile of mesosoma, distance from approximate inflection point, where downward sloping pronotum curves into anteriorly projecting neck, to posteroventral propodeal lobes (Fig. 4).

SPL Propodeal spine length; measured from tip of propodeal spine to closest point on outer rim of propodeal spiracle, maximizing spine length in lateral view (Fig. 4).

LHT Length of metatibia, excluding the proximomedial condyle (Fig. 3).

CI Cephalic index: HW/HL

OI Ocular index: EL/HL

SI Scape index: SL/HW

PTHI Petiole height index: PTH/PTL

PTWI Petiole width index: PTW/PTL

PPI Postpetiole width index: PPW/PPL

SPI Propodeal spine index: SPL/WL

LBI Leg-body index: WL/LHT

Queen measurements (in addition to worker measurements)

MSNW Mesonotal width; maximum width of mesonotum, measured in dorsal view (Fig. 7).

MSNL Mesonotal length; maximum length of mesonotum, measured in dorsal view (Fig. 7).

MSNI Mesonotal index: MSNW/MSNL.

TABLE 1. Standard ranges for size statements in this study.

\begin{tabular}{llllll}
\hline (in $\mathrm{mm})$ & HW $($ worker) & WL $(\mathrm{w})$ & SPI $(\mathrm{w})$ & HW $($ queen $)$ & WL $(\mathrm{q})$ \\
\hline Very small & $\leq 0.75$ & $\leq 0.75$ & - & - & - \\
Small & $0.75-0.90$ & $0.75-0.90$ & $\leq 0.18$ & $\leq 1.40$ & $\leq 2.30$ \\
Medium & $0.91-1.05$ & $0.91-1.10$ & $0.19-0.28$ & $1.41-1.64$ & $2.31-2.69$ \\
Large & $1.06-1.19$ & $1.11-1.24$ & $\geq 0.29$ & $\geq 1.65$ & $\geq 2.70$ \\
Very large & $\geq 1.20$ & $\geq 1.25$ & - & - & - \\
\hline
\end{tabular}

Terminology used in description of sculpture (after Harris, 1979)

Aciculate Appearing as if irregularly scratched with a needle (Fig. 8).

Areolate Divided into a number of small, irregular, impressed spaces (Fig. 9).

Carinate With one or a few longitudinal carinae, narrow raised ribs or ridges (e.g. Fig. 14).

Carinulate With several small, elevated longitudinal ridges or carinae (less prominent than carinate; not illustrated).

Costulate With longitudinal, coarse raised ribs or ridges; much coarser and extensive than carinulate (Fig. 11).

Reticulate Superficially net-like or made up of a network of lines (Fig. 10).

Rugulose Minutely wrinkled (not illustrated).

Colour images and measurements were created with a JVC KY-F75U digital camera, a Leica MZ16A stereomicroscope and Syncroscopy Auto-Montage (v5.0) software. The images of ants in full-face, dorsal and lateral view presented here are also publicly available on Antweb (www.antweb.org). Line drawings were produced by tracing colour images in Adobe Illustrator CS5. Species distributions were plotted with ArcMap (v9.3) within the software ArcGIS, based on coordinates (latitude and longitude) given on the specimen labels of all material. For material lacking this information the following sources were used to georeference 

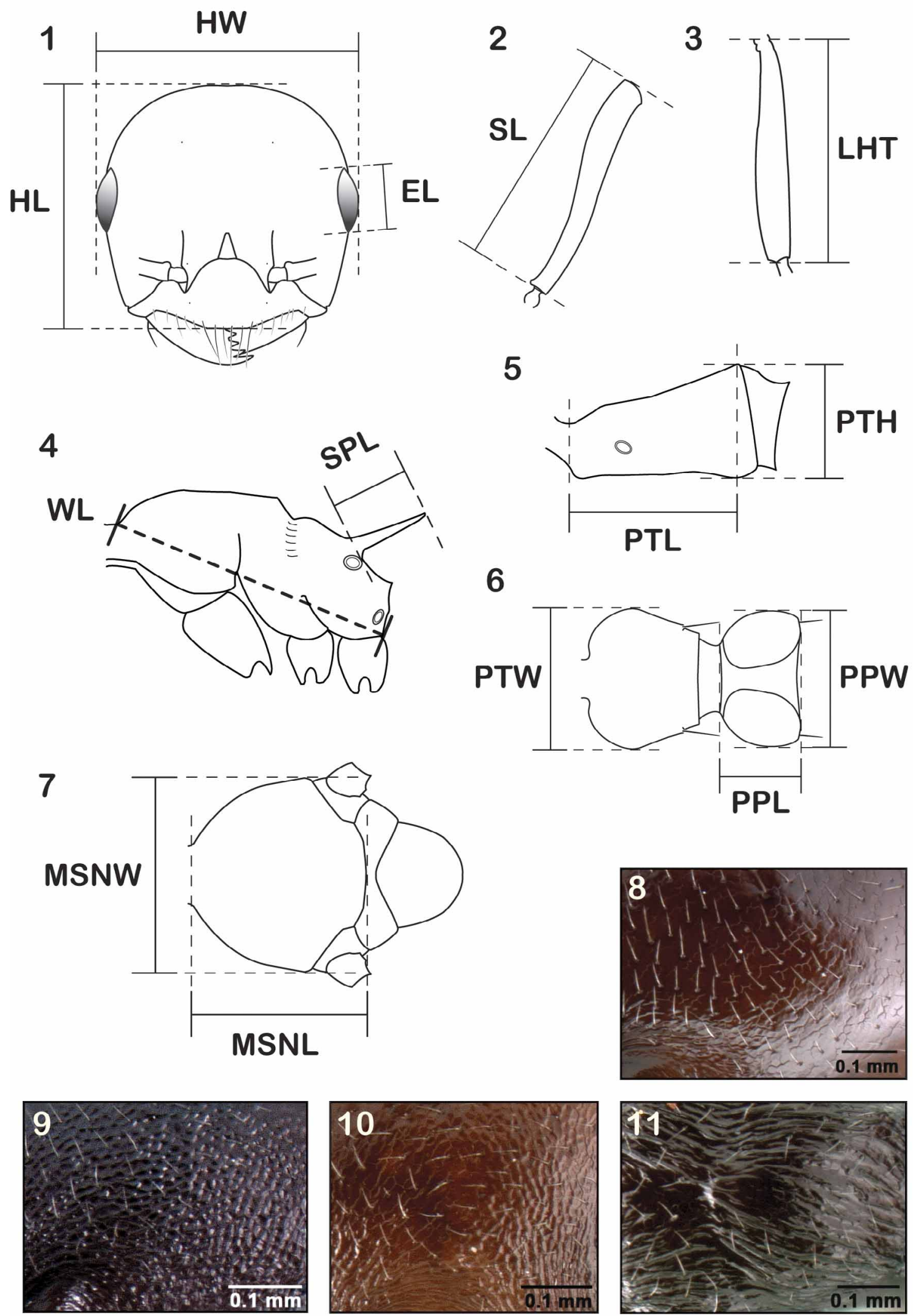

FIGURES 1-11. Measurements and sculpture patterns. 1: head width, head length and eye length; 2: scape length; 3: length of hind tibia; 4: Weber's length and spine length; 5: petiole length and petiole height; 6: petiole width, postpetiole width and postpetiole height; 7: mesonotal width and mesonotal length; 8: aciculate, 9: areolate, 10: reticulate, 11: costulate sculpture. 
collection sites: United States Board on Geographic Names (1989), Viette (1991), the GEOnet Names Server (National Geospatial-Intelligence Agency, 2010) and the Gazetteer to Malagasy Botanical Collecting Localities (Schatz \& Lescot, 2003). Classification of major geographic regions in Madagascar throughout species descriptions follows Gautier \& Goodman (2003). Common abbreviations within locality data are: P.N. $=$ Parc National, R.S. = Réserve Spéciale, F = Forêt, P.C. $=$ Parc Naturel Communautaire, R.N.I. $=$ Réserve Naturelle Intégrale.

The species described here were sequenced for fragments of one mitochondrial gene (COI) and three nuclear genes (Arginine Kinase, Long Wavelength Rhodopsin and CAD) for a total of 3120bp of DNA sequence data, using methods described in Ward \& Downie (2005) and Brady et al. (2006). From 1 to 5 individuals per species, and sixteen individuals for the species-complex were sequenced for the nuclear data. The mitochondrial data comprise 3 to 6 individuals per species and 48 individuals within the species-complex. These genetic data will be analyzed and presented in more detail elsewhere, but it is here used as important information to support species boundaries inferred from morphology and geography.

The International Commission on Zoological Nomenclature (1999) requires lectotypes designated after 1999 to "contain an express statement of deliberate designation" (amended Article 74.7.3). I use the statement 'hereby designated lectotype' to fulfill this requirement. Lectotypes have been designated where a name lacks a holotype or lectotype and unambiguous syntypes have been identified. The purpose is to provide stability of nomenclature, and designation is done in a revisionary context in agreement with the amended Recommendation 74G of Article 74.7.3. Neotype designations have further been made for names with no extant name-bearing types that are in need of a name-bearing type "to objectively define the nominal taxon" (Article 75.1, ICZN, 1999). Neotypes have been selected in agreement with the qualifying conditions stated in Article 75.3, which are considered in detail in the respective species descriptions.

\section{RESULTS}

\section{Key to the Subgenera of the Crematogaster of the Malagasy region: Workers}

1. Antennae 10-segmented...... Decacrema

Antennae 11-segmented..... . .2

2(1) Petiole in dorsal view rectangular, with four angular or acute corners (Fig. 12); very small species (HW $\leq 0.75$, WL $\leq 0.75)$.

- $\quad$ Petiole in dorsal view variable, but never rectangular; size variable......

3

3(2) Petiole in dorsal view ovo-rectangular (Fig. 13), with anterior corners rounded and posterior corners angular or acute, and dorsal face flat; dorso-lateral margins of metanotum carinate (Fig. 14) or angular (Fig. 15), with at least 1 long, flexible seta on either side; very small to small species $(\mathrm{HW} \leq 0.90, \mathrm{WL} \leq 0.90)$

Orthocrema

- Petiole shape variable, if ovo-rectangular, then dorsal face concave; dorso-lateral margins of metanotum never carinate; size variable.

4(3) Anterior margin of clypeus medially protruding as thin flange over mandibles (Fig. 18), anterior part of clypeus transversely concave; subpetiolar tooth or protrusion absent, petiolar stalk narrow (Fig. 16b); petiole shape usually more or less slender and suboval (Fig. 16a), rarely flared or oval Oxygyne

- Anterior margin of clypeus never medially protruding over mandibles (Fig. 19), anterior part of clypeus transversely convex; subpetiolar tooth or protrusion mostly present, variable in size and form, if close to absent (Fig. 17b), then petiole broadly flared anteriorly (Fig. 17a) and distinctly wider than long, never slender and suboval; otherwise petiole shape variable. Crematogaster

\section{Key to the Subgenera of the Crematogaster of the Malagasy region: Queens}

(as far as queens are known)

1 Antennae 10-segmented.

Decacrema

Antennae 11-segmented. 2

2(1) Mandibles sickle-shaped with single apical tooth (Fig. 18), very rarely with 4 teeth where apical tooth enlarged and 3 additional normal-sized teeth present; clypeus medially protruding as thin flange over mandibles (Fig. 18) 
- Mandibles broad with 4-5 teeth, apical tooth at most slightly enlarged (Fig. 19); clypeus not protruding over mandibles (Fig. 19)

3(2) Petiole in dorsal view rectangular (Fig. 12); propodeal spines absent Mesocrema

Petiole in dorsal view variable, but never rectangular; propodeal spines variable

4(3) Petiole in dorsal view ovo-rectangular (Fig. 13) and with postero-lateral denticles or tubercules; postpetiole without median impression Orthocrema

- Petiole shape variable, but never ovo-rectangular, postero-lateral denticles absent; postpetiole variable, but at least posteriorly with weak median impression Crematogaster

\section{List of Crematogaster species/subspecies currently classified under Oxygyne, Orthocrema, Mesocrema and Crematogaster and described from Madagascar}
Oxygyne
C. agnetis Forel
C. descarpentriesi Santschi
C. emmae Forel
C. emmae laticeps Forel
C. inops Forel
C. marthae Forel
C. ranavalonae Forel
C. ranavalonae paulinae Forel
C. ranavalonae pepo Forel

\section{Orthocrema}

C. madecassa Emery
Mesocrema
C. rasoherinae Forel
C. rasoherinae brunneola Forel

\section{Crematogaster}

C. adrepens Forel

C. castanea Smith

C. deegeri Forel

C. deegeri lunaris Santschi

C. kelleri Forel

C. lobata Emery

C. lobata pacifica Santschi

C. madagascariensis André

C. senegalensis Roger

C. sewellii Forel

C. sewellii marnoi Mayr

C. sewellii dentata Dalla Torre

C. sewellii improba Forel

The taxonomy of the above species will be the focus of separate studies, to be published elsewhere. 

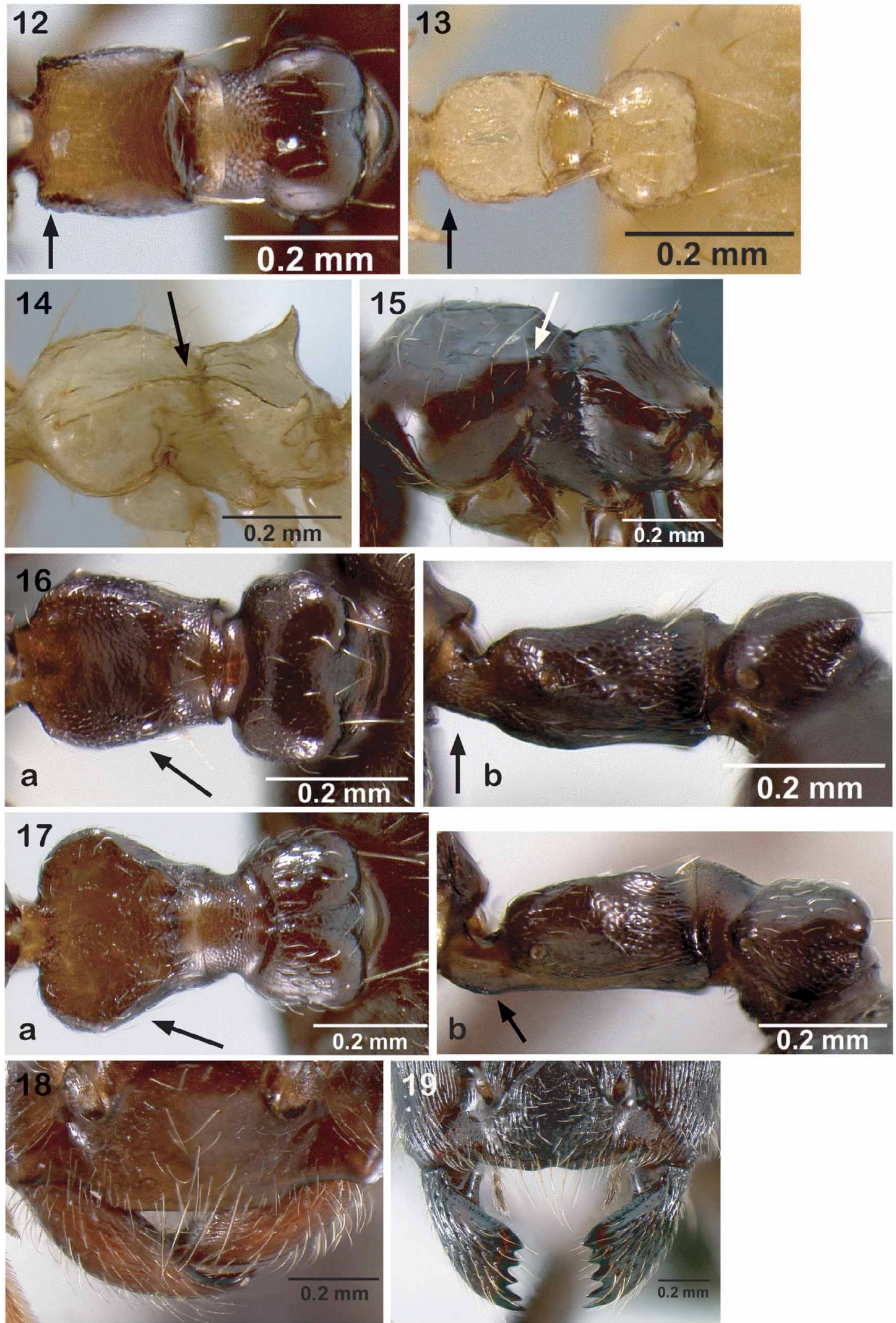

FIGURES 12-19. Key to the 'subgenera' present in Madagascar. 12: petiole in dorsal view rectangular; 13: petiole in dorsal view ovo-rectangular; 14: dorso-lateral margins of mesonotum carinate; 15: dorso-lateral margins of mesonotum angular; $\mathbf{1 6}$ a: petiole slender (in dorsal view) and $\mathbf{b}$ : petiolar stalk narrow (in lateral view); 17 a: petiole flared (in dorsal view) and b: petiolar tooth absent (in lateral view); 18: mandibles with a single apical tooth, sickle-shaped; 19: mandibles with 5 teeth, broad (this type of mandibles also often with 4 teeth). 


\section{Synopsis of the subgenus Decacrema Forel 1910}

Decacrema Forel, 1910a:18 and 1910b:9 [as subgenus of Crematogaster]. Type species: Crematogaster schencki, by subsequent designation of Wheeler, W.M. 1911:161.

Decacrema as genus: Soulié 1964:398.

Decacrema as subgenus of Crematogaster: Forel, 1910a:18; Forel, 1910b:9; Forel, 1917:242; Arnold, 1920a:547; Emery, 1922:137; Wheeler, W.M. 1922a:661; Bolton 1995:26; Bolton 2003:238; Bolton et al., 2007.

Decacrema as junior synonym of Crematogaster: Brown 1973:179 [provisional]; Smith, D.R. 1979:1376; Hölldobler and Wilson 1990:13.

[Decracrema Arnold, 1920: 547; incorrect subsequent spelling.]

\section{Species previously assigned to Decacrema and their distribution}

Seventeen nominal species of Crematogaster have traditionally been assigned to Decacrema and are listed by Bolton (1995) (note that Bolton et al. (2007) omit 3 of these species and are therefore not referenced). From South-East Asia these include Crematogaster angulosa André, C. borneensis André (incl. 8 subspecies), $C$. biformis André, $C$. captiosa Forel, $C$. cephalotes Smith, $C$. decamera Forel and $C$. enneamera Emery; from Sub-Saharan Africa C. arthurimuelleri Forel, C. edentula Santschi, C. lango Weber, C. liengmei Forel (incl. 2 subspecies), C. petiolidens Forel and C. solenopsides Emery (incl. 3 subspecies); from Madagascar C. ensifera Forel, C. grevei Forel, C. hova Forel (incl. 2 subspecies) and C. schencki Forel. Because of the likely non-monophyly of Decacrema, as currently investigated, the synopsis and diagnosis below are restricted to the species of the Malagasy clade.

\section{Taxonomic synopsis of Malagasy Decacrema}

C. ensifera Forel 1910 assigned to C. hova-complex

C. grevei Forel 1891

C. hova Forel 1887 assigned to C. hova-complex

$=$ C. hova latinoda Forel 1892 NEW SYNONYMY

C. mahery NEW SPECIES

C. malala NEW SPECIES

C. nosibeensis Forel 1891 NEW STATUS

C. sabatra NEW SPECIES

C. schencki Forel 1891 assigned to C. hova-complex

C. sisa NEW SPECIES

\section{Worker diagnosis of Malagasy Crematogaster (Decacrema)}

Size small to very large (HW 0.81-1.31, WL 0.78-1.34).

Masticatory margin of mandibles with 4-5 teeth; clypeus in lateral view always moderately convex, and with a median notch on anterior margin; frontal triangle always somewhat impressed; frontal carinae short; head always slightly wider than long; posterior margin of head straight or slightly convex, often with slight median depression; eyes normal-sized (OI 0.21-0.29) and convex; 9-14 ommatidia across long axis; antennae 10-segmented; antennal club moderately or weakly 3-segmented; antennal scapes always somewhat surpassing posterior head margin, weakly to moderately arched.

Promesonotum antero-laterally either rounded, subangular, angular or with distinct, raised shoulders; median portion of promesonotum varying from convex to weakly concave; distinctness of promesonotal suture variable, absent, incomplete or complete, but never flexible; mesonotum sometimes raised with respect to pronotum and forming minute median tubercule; promesonotum postero-laterally variable, from rounded, to broadly angular, to distinctly carinate and clearly demarcating promesonotum from propodeum in lateral view; metanotal groove shallow; dorsal face of propodeum short to absent, posterior face long and gently sloping $\left(\sim 45^{\circ}\right)$; propodeal spines always present, spiniform, small to very long (SPI 0.13-0.40), straight or curved in lateral view, parallel to strongly diverging in dorsal view; petiole shape highly variable, in dorsal 
view suboval, hexagonal or trapezoidal, always at least slightly wider than long and usually widest in anterior half, with anterolateral corners often rounded and sometimes distinctly flared; in lateral view petiole highest posteriorly, petiole dorsally flat to weakly concave, laterally rounded to distinctly carinate, in some cases with postero-lateral tubercle or denticle, petiole ventrally convex; petiole antero-ventrally with blunt, angular or rounded protuberance, or more or less acute and long tooth; postpetiole always much wider than long, bispheric and grooved or distinctly medially impressed, lacking a ventral tooth.

Sculpture costulate on mandibles, clypeus, between eyes and frontal carinae, and below eyes; rest of sculpture on head, mesosoma petiole and postpetiole highly variable, from very much reduced, aciculate or weakly reticulate, to pronounced, deeply costulate or areolate; pilosity fairly variable, clypeus with 6-12 erect long setae along and above margin; pilosity on face with $0-8$ erect setae, otherwise regular appressed pubescence; head ventrally usually with appressed pubescence throughout and interspersed scattered erect to suberect setae; promesonotum with $0-8$ erect setae, mostly in humeral region; petiole and postpetiole with or without single erect dorso-posterior setae; otherwise petiole and postpetiole with scattered appressed to decumbent pilosity; abdominal tergites and sternites 4-7 with abundant short appressed pubescence, scattered erect, longer setae usually present on sternites, sometimes also on tergites.

Colour variable; all shades of brown, orange-red, black; sometimes bi-coloured with gaster darker or lighter coloured than mesosoma.

Queen diagnosis of Malagasy Crematogaster (Decacrema) (based on queens of Crematogaster grevei, C. nosibeensis and C. hova-complex; queens of C. malala, C. mahery, C. sabatra and C. sisa are not known).

Size variable, small to very large (HW 1.26-1.97; WL 2.06-3.05).

Mandibles with 5 teeth; eyes medium sized (OI 0.29-0.37) and convex, > 20 ommatidia across longitudinal axis; distance between lateral ocelli about twice as long as between median and lateral ocelli; antennae 10-segmented; antennal scapes fairly short, not or just barely surpassing head margin; head always wider than long, head width tapering from posterior to anterior margin, head shape thus more or less triangular in full face view.

Mesosoma variable, from long and slender to short and compact; pronotal collar more than twice as wide as long; scutellum in dorsal view broadly rounded and somewhat tapering posteriorly, in lateral view projecting over postscutellum; postscutellum in lateral view weakly projecting over propodeum; dorsal face of propodeum short to very short, slope of posterior face steep, almost vertical; propodeal spines reduced, ranging from tubercules to denticles to very sharp points; petiole characters following those in workers, but less variable within species; dorsal face of petiole mostly flat to weakly convex; postpetiole with median impression reduced relative to state in workers.

Head sculpture usually as in workers; mesosomal sculpture with mesonotum and scutellum scattered aciculate; mesopleuron partly aciculate, partly costulate; postscutellum finely costulate; metapleuron costulate; petiole and postpetiole sculpture variable; clypeus with 4-5 longer and abundant shorter erect setae along and above margin, 2-4 shorter erect setae near antennal insertions, and abundant appressed to subdecument pubescence elsewhere; erect pilosity on face more abundant than in worker, 10-20 short setae, further abundant appressed pilosity on face and ventral side of head; pronotum with appressed to subdecumbent pilosity on collar; mesonotum with scattered erect pilosity, usually $>20$ setae, abundant appressed pubescence throughout; pilosity on petiole and postpetiole variable, but usually lacking erect pilosity; abdominal tergites 4-7 with scattered pilosity, and sternites 4-7 with more regular and dense short erect pilosity, otherwise regular appressed pubescence.

Colour variable, as in workers.

Comments. Non-Malagasy species assigned to Decacrema examined in the context of this revision included C. borneensis, C. captiosa, C. decamera, C. edentula, C. enneamera, C. lango, C. liengmei, $C$. petiolidens and $C$. solenopsides. I found $C$. liengmei to be morphologically very close to the Malagasy Decacrema species, both workers and queens, and this species fits well in the diagnosis given above. Features that distinguish workers of all Malagasy Decacrema species from the workers of the rest of the examined species are 1) a long scape that always surpasses the posterior head margin (versus short and not surpassing in other species examined), 2) the head being always distinctly wider than long (versus often HL > HW in other 
species examined), 3) a generally much larger body size (most examined African and Asian species with HW $<0.50$ and $\mathrm{WL}<0.55$ ), 4) larger propodeal spines and 5) an overall reduced erect pilosity. Conclusions on the status of the 'subgenus' beyond these preliminary data will require a comprehensive morphological and molecular analysis of all species assigned to Decacrema. It is worth highlighting that the type species of Decacrema is Crematogaster schencki, a Malagasy species, and hence the subgenus name would continue to denote the Malagasy Decacrema under any future possible nomenclatural change.

Distribution of Malagasy Decacrema. Decacrema species are found in forest habitats throughout Madagascar (Fig. 20).

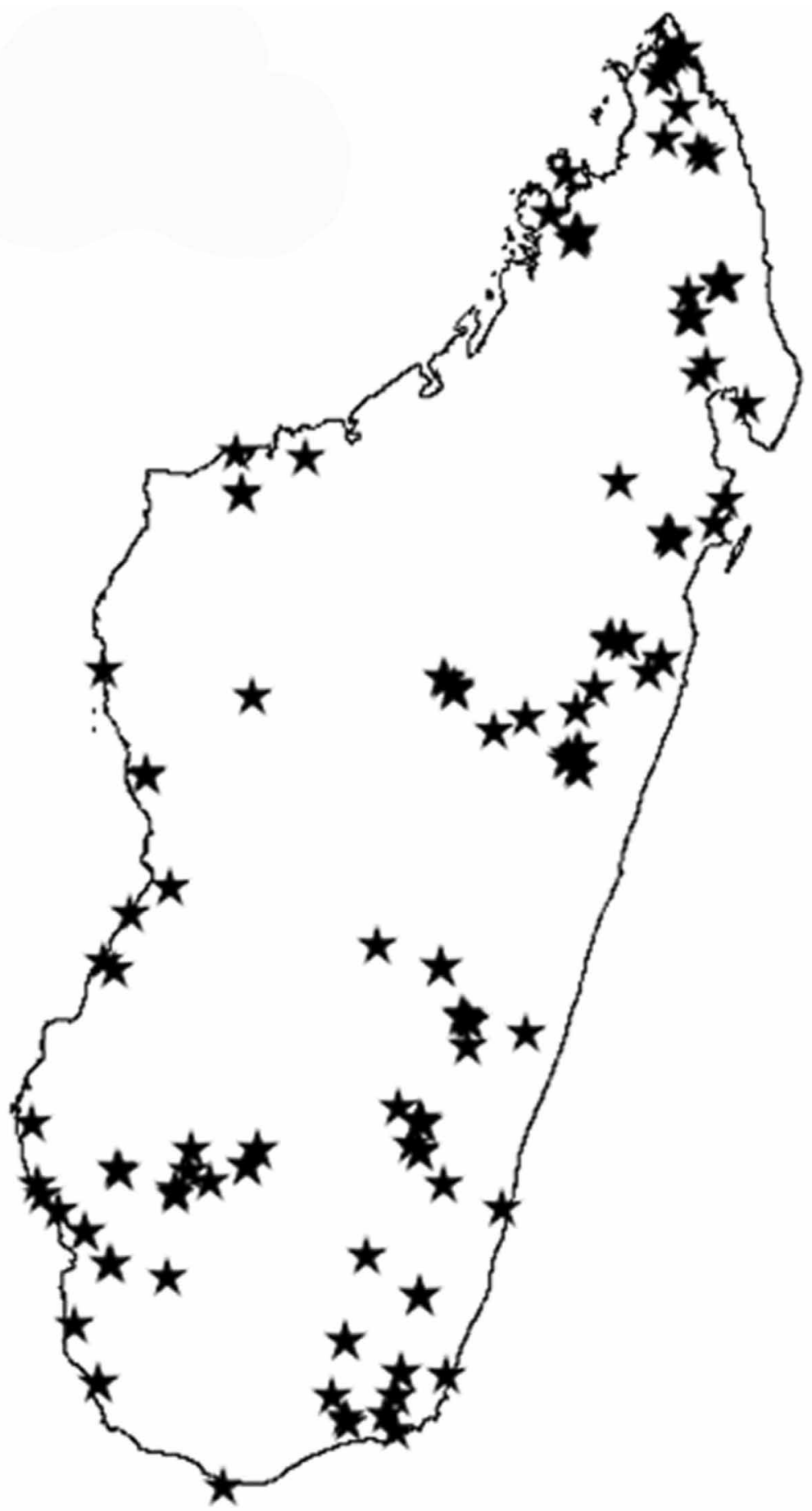

FIGURE 20. Distribution of Decacrema in Madagascar. 
$21 a$
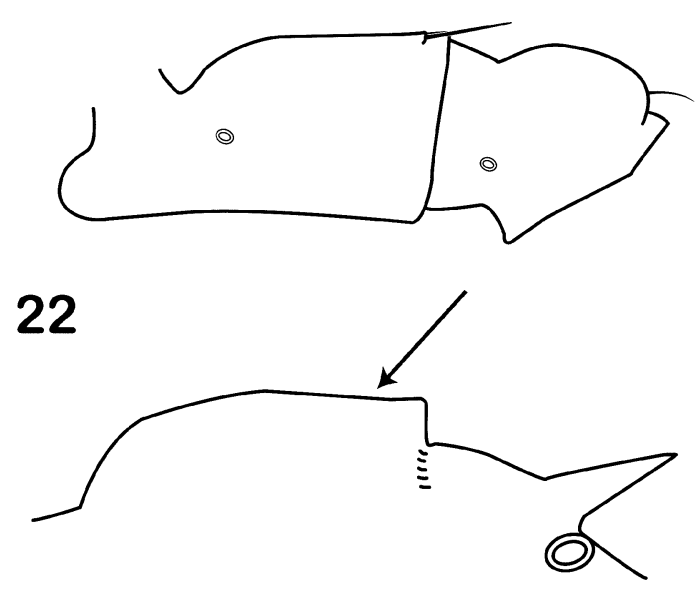

24
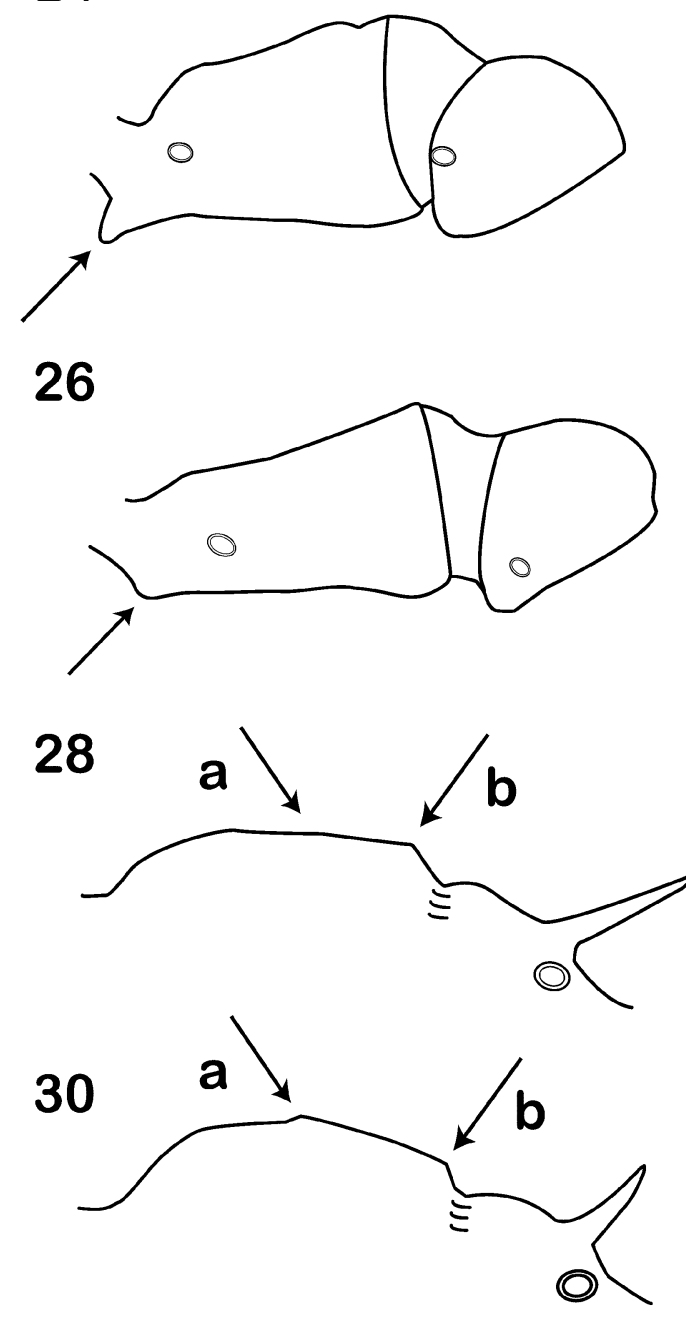

21b

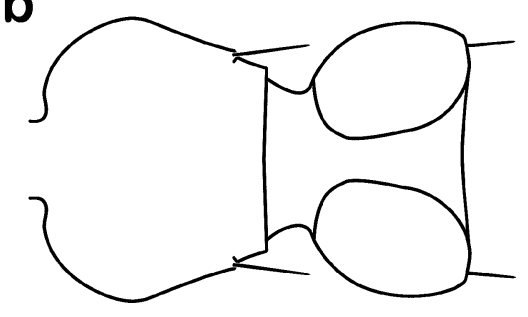

23

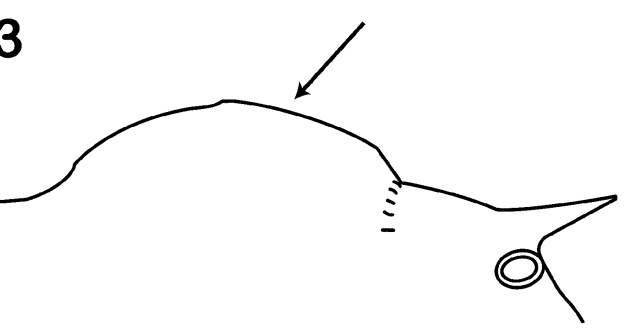

25

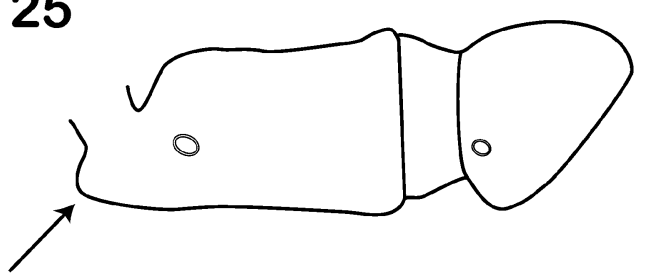

27
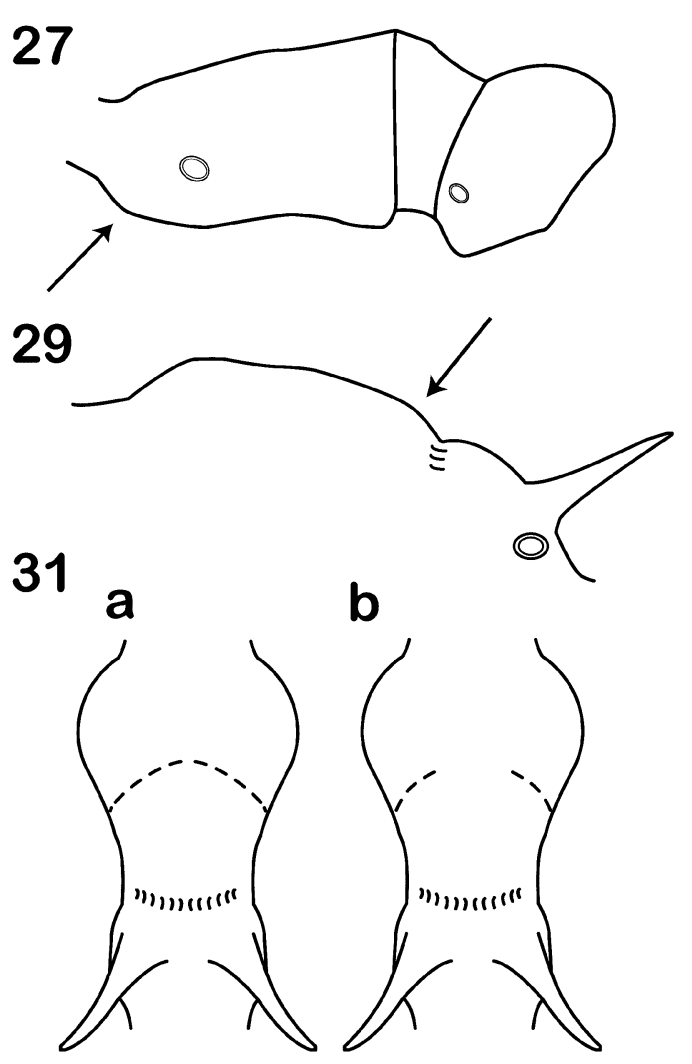

FIGURES 21-31. Key to Malagasy Decacrema. 21 a: petiole with erect setae; 21 b: postpetiole distinctly bilobed and with erect setae; 22: promesonotum in lateral view flat; 23: promesonotum in lateral view convex; 24: subpetiolar process as acute tooth; 25: subpetiolar process as broad protuberance; 26: subpetiolar process as angular dent; 27: subpetiolar process absent; 28: mesonotum a: not raised in respect to pronotum b: postero-laterally angular, 29: mesonotum rounded postero-laterally; 30: mesonotum a: raised in respect to pronotum b: postero-laterally angular; 31: promesonotal suture a: complete b: incomplete. 


\section{Key to the workers of the Crematogaster (Decacrema)-group, Malagasy region}

1. Petiole dorsally with postero-lateral tubercles, each bearing a single, stiff and long erect seta (Fig. 21a); postpetiole distinctly bilobed and broadly medially impressed, with a pair of dorso-posterior stiff, long erect setae (Fig. 21b); abdominal tergites 4-7 usually with scattered erect pilosity; mandibles with 4 teeth.

- Petiole dorsally lacking postero-lateral tubercle, setae commonly absent, if present (rare) then short and feeble; postpetiole variable, usually without erect setae; erect pilosity absent from abdominal tergites 4-7; mandibles with 4-5 teeth.....

2(1) Lateral portions of promesonotum raised with respect to median portion, and with postero-lateral carinate denticles and lateral carinae bordering metanotal groove, in lateral view promesonotum appearing dorsally flat (Fig. 22, or as in Fig. 37b); widespread in western and southern dry and spiny forests.....

C. grevei

- Lateral portions of promesonotum not raised with respect to median portion, postero-laterally rounded or angular and metanotal groove without lateral carinae, in lateral view promesonotum forming a more or less convex outline (Fig. 23, or as in Fig. 36b); rare, montane forests of southern central Madagascar...... C. malala

3(1) Head and promesonotal sculpture well pronounced, deeply costulate (Fig. 11 and 47a) or areolate (Fig. 9 and $48 \mathrm{a}$ ).. C. hova-complex (morphotypes 4,5 , and part of 3 )

- Head and promesonotal sculpture less developed, from reduced-aciculate (Fig. 8, or e.g. 44a) to reticulate (Fig. 10), reticulate-areolate (e.g. Fig. 46a) to weakly costulate .

4(3) Face lacking erect setae except a single pair near frontal carinae; subpetiolar process developed as broad protuberance (Fig. 25 and 38b); small to medium size (HW 0.86-1.04, WL 0.80-1.05); propodeal spines small to medium size (SPI 0.15-0.20); sculpture on head and promesonotum always reduced-aciculate; northern, northwestern Madagascar and Ambohitantely forest.

C. nosibeensis

- $\quad$ Face with additional erect setae (careful, could be abraded) other than those close to frontal carinae; subpetiolar process variable; size highly variable, small to very large (HW 0.74-1.31, WL 0.78-1.34); propodeal spines variable (SPI 0.17-0.40), but commonly SPI > 0.20; sculpture variable.

5(4) Subpetiolar process developed as acute, long tooth (Fig. 24 and 39b); promesonotal suture usually complete; medium to large size (HW 0.89-1.14, WL 0.91-1.12); propodeal spines small to medium size (SPI 0.17-0.21); head and promesonotal sculpture always reduced-aciculate; rare, known only from montane forests in R. S. Manongarivo

C. sisa

- Subpetiolar process variable, moderately articulated as angular dent (Fig. 26, 41b, 49b, 50b, 52b and 53b), close to absent (Fig. 27 and 51b), or rarely as a broad protuberance (Fig. 40b); promesonotal suture often incomplete, if complete, then large to very large species (HW 1.09-1.21, WL 1.18-1.32); otherwise size highly variable; propodeal spines usually with SPI >0.20; sculpture variable.

6(5) Mesonotum postero-laterally distinctly angular to tuberculate (Fig. 28b and 30b, or as in 32b-35b); large to very large species (HW 1.09-1.31, WL 1.18-1.34); head usually reduced-aciculate, sometimes reticulate to feebly costulate; uncommon, central, eastern central and southeastern Madagascar.

- Mesonotum postero-laterally rounded or slightly tuberculate (Fig. 29, and as in 44b-48b), rarely angular; body size small to large (HW 0.74-1.19, WL 0.78-1.22); head sculpture variable, aciculate, reticulate or reticulate-areolate, if head sculpture reduced and aciculate, then usually at least promesonotal sculpture more developed; widespread throughout humid forests in Madagascar..... C. hova-complex (morphotypes 1, 2, and part of 3)

7(6) Mesonotum raised with respect to pronotum and medially at least slightly tuberculate (Fig. 30a); promesonotal suture usually complete (Fig. 31a); head and promesonotal sculpture always aciculate............................. C. mahery

Mesonotum usually not raised with respect to pronotum nor medially tuberculate (Fig. 28a), promesonotal suture usually incomplete (Fig. 31b) and only laterally impressed; head sculpture and promesonotal sculpture either aciculate or reticulate to weakly costulate. C. sabatra*

* Large workers of the C. hova-complex will key out here if the character "mesonotum postero-laterally angular" is ambiguous or misassessed. Nearly all of these erroneous identifications will be resolved by considering the species distributions for C. sabatra.

\section{SPECIES ACCOUNTS}

\section{Crematogaster grevei Forel 1891}

(Fig. $37 \& 43$ )

Crematogaster grevei Forel, A. 1891:183. Syntypes, 2 workers, Morondava (Grevé) (MHNG) [examined] [The two MHNG syntypes are also imaged on AntWeb: CASENT0101572, CASENT0101558]. One syntype 
(CASENT0101572) hereby designated the LECTOTYPE.

Crematogaster (Decacrema) grevei Forel, Forel 1910a:18. Combination in Crematogaster (Decacrema).

Crematogaster (Decacrema) grevei Forel, Forel 1910b:9. Combination in Crematogaster (Decacrema).

Material examined. (BBBC, CASC, PSWC, MHNG) MADAGASCAR Fianarantsoa: P.N. Isalo: 22.29833, 45.35167, 990m (B.L.Fisher et al.); near P.N Isalo: -22.62667, 45.35817, 700-825m (R.Harin'Hala); Mahajanga: P.N. Namoroka: -16.37667, 45.32667, 100m (B.L.Fisher et al.); Mahavavy River: -16.05167, 45.90833, 20m (B.L.Fisher et al.); Rés. forest. Beanka: -18.02649, 44.05051, 250m (B.L.Fisher et al.); Morondava: -20.28333, 44.28333 (M. Grevé); 50km N Morondava: -20.06670, 44.58330 (A.Pauly), 48km ENE Morondava: -20.29650, 44.28150, 30m (D.M.Olson); 3.7km 102 ${ }^{\circ}$ ESE Belo-sur-mer: 20.73472, 44.04000, 10m (B.L.Fisher et al.); Bemeraha: -18.65000, 44.71670 (D.C.Lees); P.N. Baie de Baly: -16.01000, 45.26500, 10m (B.L.Fisher et al.); F Tsimembo-19.02139, 44.44067, 20m, -18.99528, 44.44350, 50m (B.L.Fisher et al.); Toliara: P.N. Kirindy-Mite: -20.79528, 44.14700, 80m (B.L.Fisher et al.); Kirindy: 20.04500, 44.66222, 100m (B.L.Fisher et al.); P.N. Zombitse: -22.84330, 44.71000, 770m (B.L.Fisher et al.); Isoky-Vohimena: -22.68330, 44.83330, 730m (B.L.Fisher et al.); F Vohibasia: -22.46670, 44.85000, 780m (B.L.Fisher et al.); 15km E Sakaraha: -22.90000, 44.68333, 760m (P.S.Ward); Ranobe: -23.03975, 43.61090, 30m (Frontier Project, MGF); Rés. Berenty (F Bealoka): -24.95694, 46.27150, 35m (B.L.Fisher et al.); Rés. Berenty (F Malaza): -25.00778, 46.30600, 40m (B.L.Fisher et al.); Rés. Berenty: -25.02100, 46.30550, 35m (R.Harin'Hala), -25.01670, 46.30000 (P.S.Ward); F Mahavelo: -24.75830, 46.15717, 110m, -24.75000, 46.16670, 115m (B.L.Fisher et al.); P.N. Andohahela: -24.93000, 46.64550, 300m (B.L.Fisher et al.); P.N. Andohahela: -25.01366, 46.64650, 160m (B.B.Blaimer); F Beroboka: -22.23306, 43.36633, 80m (B.L.Fisher et al.); Mahafaly Plateau: -24.65361, 43.99667, 80m (B.L.Fisher et al.); F Tsimanampetsotsa: -24.10056, 43.76000, 25m (B.L.Fisher et al.); Cap St. Marie: -25.59444, 45.14683, 160m (B.L.Fisher et al.); F Mite: 23.52417, 44.12133, 75m (B.L.Fisher et al.); Manderano: -23.52417, 44.09278, 75m (Frontier Project, MGF); Fiheranana: -23.23528, 43.87083, 65m (B.L.Fisher et al.); F Tsinjoriaky: -22.80222, 43.42067, 70m (B.L.Fisher et al.); Rés. Beza-Mahafaly: -23.65000, 44.63333, 135m (B.L.Fisher et al..), -23.68650, 44.59100, 165m (R. Harin'Hala); Rés. Beza-Mahafaly, parcel 1: -23.65000, 44.63330, 130m (P.S.Ward); Manombo: -22.81092, 43.73440, 165m (Frontier Project, MGF); F Vohidava: -24.24070, 46.28780, 500m, 24.23900, 46.28230, 850m (B.L.Fisher et al.); F Mikea: -22.90367, 43.47550, 35m (R. Harin'Hala).

Worker measurements (n=12). HW 0.82-0.89; HL 0.72-0.78; EL 0.16-0.21; SL 0.66-0.74; WL 0.791.07; SPL 0.13-0.18; PTH 0.18-0.22; PTL 0.24-0.29; PTW 0.26-0.31; PPL 0.15-0.20; PPW 0.27-0.32; LHT 0.57-0.63; CI 1.11-1.18; OI 0.21-0.27; SI 0.92-0.98; SPI 0.13-0.22; PTHI 0.74-0.79; PTWI 1.01-1.20; PPI 1.43-1.84; LBI 1.29-1.71.

Worker description. Small species (HW 0.82-0.89, WL 0.79-1.07).

Masticatory margin of mandibles with 4 teeth; posterior margin of head in full-face view with subangular corners, sometimes medially depressed; antennal scapes surpassing posterior margin of head easily; midline of eyes situated well above midline of head in full face view.

Promesonotum medially depressed and laterally with raised shoulders, these transitioning into lateral carinae and terminating in small denticles that abruptly demarcate promesonotum from propodeum; in lateral view outline of promesonotum flat; promesonotal suture absent; promesonotum without a distinct posterior face medially; propodeal spines small-medium sized (SPI 0.13-0.22), length roughly a third of the width between their bases, quickly tapering, in lateral view more or less straight, in dorsal view almost parallel and barely diverging; petiole in dorsal view suboval, with dorso-lateral margins carinate, terminating in small postero-lateral tubercules; subpetiolar process developed as broad, rounded protuberance; postpetiole distinctly bilobed and broadly medially impressed.

Head sculpture reduced aciculate to aciculate-reticulate, more pronounced in darker coloured form than in orange-red variety; mesosoma with promesonotum dorsally irregularly carinulate-reticulate; meso- and metapleuron carinulate-areolate; propodeum with dorsal face carinulate and first half of posterior face reticulate, otherwise shiny; legs shiny to reticulate; dorsal face of petiole mostly reticulate, centrally shiny; postpetiole dorsally feebly reticulate; lateral and ventral face of petiole and postpetiole coarsely reticulate; helcium dorsally shiny or reticulate, at most feebly carinulate; promesonotum with 4-8 erect humeral setae, 
rarely with 2 additional setae on lateral carinae; petiole with single stiff, long erect setae on each posterolateral tubercle; postpetiole with a pair of erect dorso-posterior setae; abdominal tergites 4-7 with scattered erect pilosity.

Two distinct colour variants: first variant distinctly bi-coloured, with pale to bright orange-red head, mesosoma, legs, petiole and postpetiole that contrast with a brown to almost black gaster (as shown in Fig. 37 and 43); second variant (to which the lectotype belongs) uni-coloured, from reddish brown to brown to dark brown, to entirely black (specimen image on Antweb: CASENT0454184).

Queen measurements (n=3). HW 1.26-1.33, HL 1.06-1.15, EL 0.36-0.39, SL 0.87-0.93, MSNW 0.921.05, MSNL 1.71-1.93, PTH 0.31-0.36, PTL 0.41-0.42, PTW 0.48-0.53, PPL 0.30-0.34, PPW 0.46-0.53, SPL $0.03-0.08$, WL 2.06-2.23, LHT 0.88-0.96, CI 1.11-1.26, OI 0.32-0.37, SI 0.76-0.83, MSNI 0.51-0.55, PTHI 0.74-0.87, PTWI 1.15-1.28, PPI 1.54-1.58, SPI 0.01-0.04, LBI 2.33-2.38.

Queen description. Small (HW 1.26-1.33, WL 2.06-2.23). With worker characters, except as follows. Antennal scapes just barely surpassing posterior margin of head; eyes situated at midline of head in full face view; posterior margin of head straight.

Mesosoma slender (MSNI 0.51-0.55, WL 2.06-2.23); mesoscutum in dorsal view oval, about half as wide as long; mesopleuron with episternal groove carinulate to broadly carinate; in lateral view mesepisternum meeting pronotum at an oblique angle; postscutellum with posterior face rounded to flattened; propodeal suture deep and laterally reaching until level of propodeal spiracle; dorsal face of propodeum short, about one third of the size of posterior face; propodeal spines reduced, ranging from tubercule to denticles to very sharp points; petiole and postpetiole as in worker, but less prominently shaped and dorso-posterior tubercule on petiole lacking; antero-ventral subpetiolar tooth present, but reduced with respect to worker.

Propodeum with vertical carinulae on dorsal and posterior face; petiole reticulate throughout; postpetiole feebly reticulate throughout; face with 10-12 erect setae and abundant appressed pilosity; mesonotum with 20-26 erect setae; petiole and postpetiole with dorso-posterior setae and further with appressed pilosity throughout.

Colour brown to dark brown, or orange-red with gaster dark brown or black.

Comments. This species is recognizable by a combination of the following characters. The lateral portions of the promesonotum are raised with respect to the median portion and end in postero-lateral carinate denticles. Lateral carinae border the metanotal groove. The petiole has postero-lateral tubercles dorsally that each bear a single, stiff and long erect seta; the postpetiole is distinctly bilobed and broadly medially impressed, and also bears a pair of dorso-posterior stiff, long erect setae. Further noteworthy are the short and straight spines, the erect pilosity on abdominal tergites $4-7$, and the mandibles that possess only 4 teeth. $C$. grevei is strongly supported by both nuclear and mitochondrial molecular data.

Both syntype specimens in the MHNG correspond well with the brown and black coloured variants in the CASC material, and with the type description. One of the MHNG syntypes carries matching locality data and collector information with the type description and is hence chosen as the lectotype.

Variation. Apart from colouration, the two variants of C.grevei exhibit slight variations in head sculpture. Some size polymorphism is exhibited in the workers of this species. Larger workers that have a disproportionately larger mesosoma than head are often found among colony samples of normal-sized workers.

Distribution and biology. Crematogaster grevei occurs throughout the seasonally dry forests and spiny forests of western and southwestern Madagascar (Fig. 54). Among members of the Malagasy Decacremagroup, these habitats are almost exclusively inhabited by $C$. grevei, its range is parapatric or allopatric in regard to most other species in the group. Narrow sympatry exists with the $C$. hova-complex in the Isalo Range, where isolated pockets of dry forest occur at higher elevations, in remnants of western subhumid forest in the P.N. Zombitse, and in the "tsingy" of P.N. Namoroka. In the Andohahela Range C. grevei occurs at low elevation in drier habitats, and the $C$. hova-complex and $C$. mahery take over at higher elevation in the humid forest. Fisher et al. frequently collected this species nesting in dead twigs on and above the ground, in rotten logs, from leaf litter samples, and also found it occasionally nesting under stones or in stems of live plants. Carton nesting has not been recorded for this species. The absence of this behaviour is probably related 
to the drier habitat of this species, as rotting plant material here may simply lose moisture too quickly to be implemented in carton fabrication.

The two colour variants of $C$.grevei have nearly allopatric distributions. The nature of these distributions suggests that the orange variant is associated with the spiny forests of the far south and southwest parts of Madagascar, while the brown or black variant is adapted to western dry and western subhumid forest habitats. Very likely some environmental variables play a role in stabilizing this colour dimorphism, at the moment however too little is known about the ecology of the species to make further assumptions.

\section{Crematogaster hova-complex}

(Fig. 44-53)

Crematogaster hova Forel, 1887:387. Worker syntypes from Madagascar: Bois de l'Ivondro près de Tamatave (Dr.C.Keller) [MHNG, examined;]. One worker syntype (CASENT0101804, top specimen of 3w on one pin, image on Antweb) hereby designated the LECTOTYPE.

Crematogaster hova Forel, Forel,1910b:9. Combination in C. (Decacrema).

= Crematogaster hova latinoda Forel, 1892:535. Worker syntypes from Madagascar, Mangoroufer, Est Imerina (M.Sikora) [MHNG, examined]; Wheeler, 1922b:1026. Combination in C. (Decacrema). NEW SYNONYMY

Crematogaster schenki Forel,1891:182. Workers syntypes from Madagascar: Imerina: Antananarivo, Andrangoloaka (Rév. Père Camboué, Hildebrandt, Sikora) [MHNG, examined]. One worker syntype (CASENT0101748, top specimen of 2w on one pin, image on Antweb) hereby designated the LECTOTYPE.

Crematogaster schenki Forel, Forel,1910a:18 and Forel, 1910b:9. Combination in C. (Decacrema).

Crematogaster schencki as justified emendation, Emery, 1922c:138.

Crematogaster (Decacrema) ensifera Forel,1910a:18. Worker syntypes from Madagascar: Forêts vierge de Sahana [MHNG, examined]. One worker syntype (CASENT0101790, middle specimen of $3 \mathrm{w}$ on one pin, image on Antweb) hereby designated the LECTOTYPE.

Material examined. (BBBC, CASC, PSWC, MHNG, NHMB, ZMBH, ZSM) MADAGASCAR: Antsiranana: Sakalava Beach: -12.26278, 49.39750, 10m (R. Harin'Hala); 3km W of Sakalava beach: 12.28617, 49.36667, 40m (R. Harin'Hala); Montaigne Français: -12.32500, 49.33333, 150m (R. Harin'Hala); 5km SW Ambohitra [Joffreville]: -12.53330, 49.16670, 1100m (P.S.Ward); 7 km N Joffreville: -12.33333, 49.25000, 360m (R. Harin'Hala); R.S. Ambre:-12.46889, 49.24217, 325m (B.L.Fisher et al.); P.N. Montagne d'Ambre: -12.53444, 49.17950, 925m; -12.51444, 49.18139, 960m; -12.51667, 49.18333, 975m; -12.58132, 49.13368, 1110m; -12.52028, 49.17917, 1125m; -12.58500, 49.15147, 1250m (B.L.Fisher et al.); P.N. Montagne d'Ambre: -12.51670, 49.16670, 1100m (D.M.Olson); R.S. Manongarivo: -13.93145, 48.45143, 360m; -13.96167, 48.43333, 400m; -13.97667, 48.42333, 780m; -13.99833, 48.42833, 1175m; -14.02167, 48.41833, 1580m; -14.04667, 48.40167, 1860m (B.L.Fisher et al.); R.S. Manongarivo: -13.93145, 48.45183, 360m; -13.93153, 48.45213, 370m; -13.93188, 48.4492333, 470m; -13.93155, 48.449333, 520m; (B.B.Blaimer); Ampasindava, Ambilanivy: -13.79861, 48.16167, 600m (B.L.Fisher et al.); F Andavakoera: 13.11833, 49.23000, 425m (B.L.Fisher et al.); F Antsahabe: -13.21167, 49.55667, 550m (B.L.Fisher et al.); Rés. Analamerana: -12.80467, 49.37383, 225m (B.L.Fisher et al.); F Binara: -13.25500, 49.61667, 375m; 13.26333, 49.60333, 650-800m; -13.26333, 49.60000, 1100m (B.L.Fisher et al.); Montagne d'Akirindro: 15.28833, 49.54833, 600m (B.L.Fisher et al.); $6.9 \mathrm{~km}$ NE Ambanizana: $-15.56667,50.00000,825 \mathrm{~m}$ (B.L.Fisher et al.); Nosy Mangabe: $-15.5000,49.7667,300 \mathrm{~m}$ (P.S.Ward); Rés. Anjanaharibe-Sud: -14.75000, 49.50000, 875m; -14.75000, 49.46667, 1180-1280m; -14.75000, 49.45000, 1480-1585m (B.L.Fisher et al.); Montagne d'Anjanaharibe: -15.18833, 49.61500, 470-1100m (B.L.Fisher et al.); P.N. Marojejy: -14.43333, 49.78333, 450m; -14.43817, 49.77400, 488m; -14.43500, 49.76000, 775m; -14.44333, 49.74333, 1325m; 14.44500, 49.74167, 1575m; -14.44500, 49.73500, 2000m (B.L.Fisher et al.); P.N. Marojejy [Manantenina]: -14.43667, 49.77500, 450m; -14.43333, 49.76667, 750m; -14.43333, 49.75000, 1225m; -14.45000, 49.73333, 1875m (B.L.Fisher et al.); F Betaolana: -14.52996, 49.44039, 880m (B.L.Fisher et al.); Antananarivo: NE Andranomay: -18.47333, 47.96000, 1300m (B.L.Fisher et al.); F Analamazoatra: -18.38466, 48.41270, 980m (B.L.Fisher et al.); R.S. Ambohitantely: -18.19800, 47.28150, 700m; -18.22528, 47.28683, 1410m (B.L.Fisher et al.); R.S. Ambohitantely: -18.21656, 47.28275, 1520m; -18.21722, 47.28470, 1550m; - 
18.19473, 47.28642, 1560m; -18.19442, 47.28623, 1590m; -18.17272, 47.28023, 1610m (B.B.Blaimer); 25km NNE Ankazobe: -18.10000, 47.18330, 1500m (P.S.Ward); Kaloy: -18.58998, 47.65102, 1420m (B.L.Fisher et al.); Antananarivo: -18.91667, 47.51667 (Camboué); Andrangoloaka: -19.03333, 47.91667 (Sikora); Fianarantsoa: F Atsirakambiaty: -20.59333, 46.56333, 1550m (B.L.Fisher et al.); P.C. Ankazomivady: -20.77667, 47.16500, 1780m (B.L.Fisher); P.N. Isalo: -22.48167, 45.46167, 725m; 22.62667, 45.35817, 700-825m (B.L.Fisher et al.); 9km NNW Ranohira, P.N. Isalo: -22.48330, 45.38330, 800m (P.S.Ward); F Analalava: -22.59167, 45.12833, 700m (B.L.Fisher et al.); P.N. Ranomafana: -21.26650, 47.42017, 1020m; -21.29000, 47.43333, 1100m; -21.23333, 47.36667, 1110m; -21.25000, 47.36667, 1130m; -21.23667, 47.39667, 1150m (B.L.Fisher et al.); 3km W Ranomafana, nr Ifanadiana: -21.25000, 47.41670, 950m (P.S.Ward); ); 3km WNW Ranomafana, nr Ifanadiana: -21.25000, 46.43330, 840m (P.S.Ward); F.C. Vatovavy: -21.40000, 47.94000, 175m (B.L.Fisher et al.); 2km W Andrambovato: -21.51167, 47.41000, 1075m (B.L.Fisher et al.); Tsaranoro: -22.08317, 46.77400, 975m (B.L.Fisher et al.); 45km S Ambalavao, Rés. Andringitra [=P.N. Andringitra]: -22.21667, 47.01667, 720-785m (B.L.Fisher); 40km S Ambalavao, Rés. Andringitra [=P.N. Andringitra]: -22.21667, 46.96667, 1275m (B.L.Fisher); P.N. Andringitra: -22.21167, 46.84500, 1800m (S. Razafimandimby) P.N. Andringitra: -22.22350, 47.01176, 780m; -22.22806, 47.00335, 890m; -22.21495, 46.96890, 1400m (B.B.Blaimer); R.S. Ivohibe: -22.47000, 46.96000, 900m; -22.42167, 46.89833, 1200m (B.L.Fisher et al.); F Vevembe: -22.79100, 47.18183, 600m; Mahajanga: F Telomirahavavy: -18.12167, 47.20627, 1520m (B.L.Fisher et al.); F Andranorovitra: -18.11243, 47.19757, 1490m (B.L.Fisher et al.); F Sohisaka: -18.10322, 47.18692, 1460m (B.L.Fisher et al.); Rés. Marotandrano: 16.28322, 48.81443, 865m (B.L.Fisher et al.); P.N. Namoroka: -16.37667, 45.32667, 100m (B.L.Fisher et al.); Toamasina: P.N. Mananara-Nord: -16.45500, 49.78750, 225m (B.L.Fisher et al.); Rés. Ambodiriana: 16.67233, 49.70117, 125m (B.L.Fisher et al.); Forêt vierge de Sahana: -16.08333, 49.63333 (unknown); RS Ambatovaky: -16.81739, 49.29402, 360m; -16.81620, 49.29202, 425m; -16.77550, 49.26427, 430m; 16.77274, 49.26551, 450m; -16.81209, 49.29216, 460m; -16.77020, 49.26638, 470m; -16.76912, 49.26704, 475m; -16.80561, 49.29507, 480m; -16.76330, 49.26692, 520m (B.L.Fisher et al); P.N. Andasibe: -18.77071, 48.43164, 995m; -18.92639, 48.40783, 1025m (B.L.Fisher et al.); 1km SSW Andasibe [Périnet]: -18.93330, 48.41670, 920m (P.S.Ward); 6km ESE Andasibe [Périnet]: -18.95000, 48.46670, 900m (P.S.Ward); 8km ESE Andasibe [Périnet]: -18.95000, 48.50000, 800m (P.S.Ward); Rés. Perinet-Analamazoatra: -18.93330, 48.43330, 950m (D.M.Olson); F Torotorofotsy: -18.87000, 48.34670, 1070m (B.L.Fisher et al.); F Didy: 18.19833, 48.57833, 960m (B.L.Fisher et al.); Manakambahiny: -17.75000, 48.71667 (B.L.Fisher et al.); F Ambatovy: -18.84770, 48.29600, 1000m; -18.84950, 48.29470, 1010m; -18.85800, 48.28480, 1040m; 18.85083, 48.32000, 1075m; -18.83930, 48.83930, 1080m (B.L.Fisher et al.); F Analamay: -18.80623, 48.33707, 1068m (B.L.Fisher et al.); F.C. Sandranantitra: -18.04833, 49.09167, 450m (B.L.Fisher et al.); P.N. Mantadia: -18.79167, 48.42667, 875m; -18.79167, 48.42667, 895m (B.L.Fisher et al.); P.N. Zahamena: 17.75244, 48.85320, 760m; -17.75257, 48.85725, 765m; -17.75908, 48.85468, 780m (B.L.Fisher et al.); Rés. Betampona: -17.92400, 49.19967, 390m; -17.91801, 49.20074, 500m; -17.88667, 49.20250, 520m (B.L.Fisher et al.); Est-Imerina: Mangoro-ufer (Sikora); Bois de l'Ivondro près de Tamatave: -18.23333, 49.36667 (Keller); Toliara: R.S. Ambohijanahary: -18.26667, 45.40667, 1050m (B.L.Fisher et al.); F Vohimena: -22.68330, 44.83330, 730m (B.L.Fisher et al.); P.N. Zombitse: -22.84333, 44.71000, 770m ; 22.84050, 44.73117, 825m; -22.88650, 44.69217, 840m (B.L.Fisher et al.); 15km E Sakaraha: -22.90000, 44.68330 760m, (P.S.Ward); F Analavelona: -22.67500, 44.18667, 1050m; -22.67500, 44.19000, 1100m; 22.64333, 44.17167, 1300m (B.L.Fisher et al.); Fiherenana: -23.23528, 43.87083, 50m; -23.17694, 43.96083, 100m (B.L.Fisher et al.); Mahafaly Plateau: -24.65361, 43.99667, 80m (B.L.Fisher et al.); P.N. BefotakaMidongy: -23.84080, 46.95750, 1250m (B.L.Fisher et al.); P.N. Andohahela: -24.75850, 46.85370, 275m; and 24.76389, 46.75167, 900m (B.L.Fisher et al.); 10km NW Enakara, Rés. Andohahela: -24.56667, 46.81667, 430m; 13km NW Enakara, Rés. Andohahela: -24.55000, 46.80000, 1150-1250m (B.L.Fisher); P.N. Andohahela: -24.73732, 46.83950, 550m (B.B.Blaimer); 5km NNW Isaka-Ivondro, Rés. Andohahela: 24.75000, 46.85000, 280m (P.S.Ward); 6km SSW Eminiminy, Rés. Andohahela: -24.75000, 46.78330, 500m (P.S.Ward); 9km SSW Eminiminy, Rés. Andohahela: -24.73330, 46.80000, 330m (P.S.Ward); Col de Manangotry: -24.73330, 46.85000, 1500-1800m (D.M.Olson); F Ivohibe: -24.56170, 47.20020, 650m 
(B.L.Fisher et al.); Grand Lavasoa: -25.08770, 46.74900, 450m (B.L.Fisher et al.); province unknown: Central Madagascar (Hildebrandt); Südcentral Madagascar (unknown).

Worker measurements (n=58). HW 0.74-1.19; HL 0.69-1.08; EL 0.15-0.27; SL 0.65-0.93; WL 0.781.22; SPL 0.19-0.44; PTH 0.15-0.24; PTL 0.22-0.38; PTW 0.22-0.39; PPL 0.14-0.23; PPW 0.20-0.33; LHT 0.64-0.97; CI 1.04-1.19; OI 0.18-0.29; SI 0.83-0.99; SPI 0.19-0.40; PTHI 0.52-0.86; PTWI 0.83-1.39; PPI 1.24-1.79; LBI 1.17-1.56.

Worker description. Size highly variable, small to very large (HW 0.74-1.19, WL 0.78-1.22), commonly medium to large size.

Masticatory margin of mandibles with 4 teeth in smaller individuals, 5 teeth in larger specimens; posterior margin of head straight but sometimes medially depressed, laterally forming round or subangular corners; antennae with scapes reaching or surpassing posterior margin; midline of eyes situated at midline of head in full face view.

Pronotum laterally with distinct rounded or subangular shoulders; promesonotal suture incomplete, laterally impressed; outline of promesonotum in lateral view more or less rounded; dorsal face of mesonotum either flat or weakly convex, sometimes medially slightly raised over pronotum and rarely forming a small median tubercle anteriorly; lateral margin of mesonotum from rounded to slightly angular, sometimes bearing postero-lateral angular tubercules; length and divergence of propodeal spines variable; petiole highly variable; shape in dorsal view from weakly to strongly lobed, trapezoidal, hexagonal or suboval, in lateral view petiole usually tapering in height from posterior to anterior; subpetiolar process variable, either narrow to broad protuberance, with more or less angular tooth or absent, postpetiole in dorsal view from weakly to much wider than long (PPI 1.24-1.79).

Head sculpture variable (see below); sculpture on mesosoma following mostly that on head, aciculate, reticulate, costulate or areolate; dorsal face of petiole mostly shiny, ventrally coarsely reticulate; postpetiole dorsally and laterally rugulose or reticulate, helcium carinulate; face with 4-6, sometimes up to 10 erect setae; $0-2$, sometimes 4 , rarely 6 erect-suberect humeral setae present on promesonotum; petiole and postpetiole devoid of long erect pilosity, sometimes shorter, suberect setae present on postpetiole.

Colour light to dark brown or black, sometimes dorsum of $4^{\text {th }}$ abdominal segment yellow and gaster thereby bi-coloured.

Queen measurements (n=16) HW 1.60-1.97, HL 1.37-1.63, EL 0.43-0.53, SL 0.99-1.19, MSNW 1.221.60, MSNL 2.36-2.67, SPL 0.02-0.13, WL 2.68-3.05, PTH 0.38-0.45, PTL 0.47-0.59, PTW 0.53-0.69, PPL 0.40-0.47, PPW 0.54-0.68, LHT 1.08-1.36, CI 1.14-1.25, OI 0.29-0.37, SI 0.69-0.78, MSNI 0.52-0.66 , SPI 0.01-0.05, PTHI 0.70-0.91, PTWI 1.00-1.36, PPI 1.28-1.69, LBI 2.13-2.32.

Queen description. Large to very large (HW 1.60-1.97, WL 2.68-3.05). With worker characters, except as described below.

Antennal scapes usually not surpassing posterior margin of head; midline of eyes situated at or slightly below midline of head in full face view; posterior margin of head straight or medially depressed.

Mesosoma broad and long (MSNI 0.52-0.66, WL 2.68-3.05); mesoscutum in dorsal view oval, less than half as wide as long; mesopleuron with episternal groove carinulate and usually ending in small pit just short of margin; in lateral view mesepisternum meeting pronotum at an oblique angle; postscutellum with posterior face distinctly flattened; propodeal suture weak, laterally not reaching level of propodeal spiracle; dorsal face of propodeum short, about one third of the size of posterior face; propodeal spines reduced, ranging from tubercule to denticles to very sharp points; petiole shape usually triangular, tapering from anterior to posterior margin, antero-ventral subpetiolar tooth absent.

Propodeum with horizontal carinulae on dorsal face; petiole dorsally more or less shiny, laterally and ventrally reticulate to costulate; postpetiole aciculate throughout; face with 10-20 short, erect setae, and otherwise abundant appressed pilosity; mesonotum with scattered erect pilosity, usually $>20$ setae, and abundant appressed pubescence; petiole laterally with short suberect pilosity, dorso-posterior erect setae absent; postpetiole with dorso-posterior erect setae present, further scattered suberect pilosity may be present; petiole and postpetiole with appressed pilosity throughout.

Colour light to dark brown, or black; metasoma usually lighter coloured. 
Variation. I distinguish 5 morphotypes of workers within the $C$. hova-complex on the basis of sculpture, body size and propodeal spines. Designation of these morphotypes is somewhat arbitrary as they are not entirely distinct from each other, but have intermediate forms - and further intermediate forms of these 'intermediates' exist. They should therefore not be perceived as distinct units, but rather as samples from an overall gradient. For the purpose of distribution mapping some ambiguous assignments to morphotypes were therefore made. The following distinction however simplifies the description of morphological variation greatly, and allows for its analysis for possible geographic patterns. Moreover, this morphotype concept presents a starting hypothesis for a much needed study on the population genetic level of the C. hovacomplex.

Morphotype 1 (mt1) (Fig. 44 \& 49). Small to large size (HW 0.83-1.10, WL 0.87-1.14). Head sculpture reduced aciculate to superficially areolate; propodeal spines medium size, at most as long as distance between their bases (SPI 0.21-0.28), in dorsal view only weakly diverging $\left(<20^{\circ}\right)$. Colour light brown to dark brown, less common black, metasoma often lighter coloured.

This morphotype conforms most closely with the $C$. hova and $C$. hova latinoda type specimens. $C$. hova latinoda type material has a petiole that is strongly broadened anteriorly with respect to C. hova.

Morphotype 2 (mt2) (Fig. 45 \& 50). Medium to large size (HW 0.92-1.19, WL 0.93-1.22). Head sculpture reticulate, reticulate-areolate to weakly areolate; propodeal spines medium size, at most as long as distance between their bases (SPI 0.22-0.26), in dorsal view only weakly diverging $\left(<20^{\circ}\right)$. Colour light brown to black, metasoma often lighter coloured in brown form, $4^{\text {th }}$ abdominal segment or entire gaster sometimes of yellow coloration (may be due to parasitism rather than inherent pigmentation).

This morphotype is intermediate in regard to $\mathrm{mt} 1$ and $\mathrm{mt} 4$.

Morphotype 3 (mt3) (Fig. 46 \& 51). Small to large size (HW 0.74-1.13, WL 0.78-1.19). Head sculpture from scattered aciculate to reticulate to costulate-areolate or areolate; propodeal spines large and robust, often "swollen" at base, at least as long or longer than distance between their bases (SPI 0.29-0.40), in dorsal view strongly diverging, ca. $>30^{\circ}$ from lateral margin of propodeum. Colour brown to black.

This morphotype conforms best with the $C$. ensifera type material.

Morphotype 4 (mt4) (Fig. 47 \& 52). Medium to large size (HW 0.97-1.18, WL 1.01-1.21). Head sculpture deeply costulate to costulate-areolate; propodeal spines medium size (SPI 0.24-0.29), about as long as distance between bases or shorter, in dorsal view weakly or moderately diverging $\left(<30^{\circ}\right)$. Colour brown to black, more often black, sometimes gaster shows bi-coloration described for $\mathrm{mt} 2$.

This morphotype conforms closely to the $C$. schencki type material. Together with $\mathrm{mt} 2$ it is the most common morphotype of the hova-complex.

Morphotype 5 (mt5) (Fig. 48 \& 53). Medium to large size (HW 0.96-1.13, WL 1.01-1.17). As mt4, except as follows. Head sculpture deeply areolate. Colour black.

Comments. The question whether the $C$. hova-complex consists of one single, highly variable species, or multiple, very closely related species was thoroughly investigated in this study, with molecular data adding an important second line of evidence. Both nuclear and mitochondrial data were inconclusive in regard to the status of the involved taxon or taxa. Phylogenetic analyses of sequence data show two distinct "clusters" among specimens sampled within the complex. These form a clade together with $C$. sabatra, but in consequence of the exclusion of the latter from the C. hova-complex (see species description of $C$. sabatra) are a paraphyletic grouping by themselves. The two clusters further only broadly correspond with the characteristics of the morphotypes, in that $\mathrm{mt} 1+\mathrm{mt} 5$ group together in one cluster, and $\mathrm{mt} 3+\mathrm{mt} 4$ group together in the other. Morphotype 2 however appears in both clusters. A geographic pattern, as would be expected if the two clusters represented geographically separated regions with reduced gene flow, also could not be discerned. The underlying cause of this problematic species-complex may be one of incipient speciation where complete reproductive isolation is still lacking between diverging populations within a species, or alternatively one of hybridization between previously well separated species. Cases like this are discovered more and more in ants (e.g. Feldhaar, 2008; Korczynska et al., 2010), and hybridization has already been posited to occur in members of the subgenus Crematogaster (Decacrema) from Asia (Feldhaar et al., 2010). 
Consequently three described species are here placed under the C. hova-complex: C. hova, C. schencki and $C$. ensifera. The identities of these species have been thoroughly investigated, including examining the type specimens in the MHNG collection. Among the material examined for this study, specimens close to the type localities also closely resemble Forel's type specimens - material from the vast remainder of the distribution range however presents a variety of intermediate morphological forms. Crematogaster hova latinoda is synonymised under $C$. hova since its syntype material very closely resembles that of $C$. hova in all but the width of the petiole and postpetiole, which are - the name implies - wider in C. hova latinoda than in C. hova.

Forel (1887) described C. hova first, with a very detailed species description. When he subsequently described $C$. schencki (Forel, 1891, here originally misspelled as C. schenki) he separated this new species from $C$. hova solely by size and sculpture - however, he already suspected a strong relatedness between the two species and concluded with the thought $C$. schencki might only be a "race" of $C$. hova. Two decades later Forel described $C$. ensifera as being very close to $C$. hova and $C$. schencki, distinguishing it from both by petiole shape and greater spine length, from $C$. hova by greater size and robustness and from $C$. schencki by sculpture and colour (Forel, 1910).

In conclusion, Forel's taxonomic decisions were based upon a set of highly variable morphological characters - and an incomplete sample of specimens from very few localities in Madagascar. Inevitably, extensive sampling and more sophisticated taxonomic methods here have presented a much more complex picture. These advances have certainly been able to better define this taxonomic problem, even though a definite solution on the species level is not attained at this point in time. For the time being the names C. hova, $C$. schencki and $C$. ensifera should thus be treated as part of and referred to as the C. hova-complex. An alternative possibility could be to use C.(hova) as a "species aggregate" (Article 6.2, ICZN, 1999) for the three names, as has been proposed by Taylor (1989 and 1991) for similar cases in ants.

Distribution and biology. The $C$. hova-complex has a wide distribution throughout the humid and transition forests of northern, central and southeastern Madagascar. It is generally absent from the western dry deciduous and spiny forests occupied by $C$. grevei, but present in isolated pockets of humid or transition forest remaining at a few locations in the west, such as in e.g. P.N. Zombitse or P.N. Isalo (see Fig. 60). From the very northern tip to the extreme south of the island these ants are a prominent element of the rainforest canopy fauna, inhabiting all altitude levels, with a highest record of $2000 \mathrm{~m}$ in the Marojejy massif. The C. hovacomplex occurs in sympatry with C. malala, C. sabatra, C. sisa, C. mahery and C. nosibeensis, and a narrow sympatry or parapatry exists with $C$. grevei (see details under description of $C$. grevei).

The distribution of the five morphotypes can be characterized as follows. Mt1 is found predominantly in the far north, along the east coast and throughout the above mentioned remnant rainforests in the west of Madagascar (Fig. 61). This morphotype co-occurs mostly with $\mathrm{mt} 2$, and only rarely with the other morphotypes. Mt2 occurs over the entire range of the complex, but with an emphasis on the northern and central highland localities (Fig. 62), and further widely co-exists with all other morphotypes. Mt3 has a distinct distribution along the east coast and eastern central highland localities, with isolated distributions in the Sambirano Region of the northwest and in the very far north (Fig. 63). This morphotype co-occurs widely with $\mathrm{mt} 2$, but is mostly allopatric with $\mathrm{mt} 4$. Mt4 is the most abundant form of the complex and is found throughout the north, the highlands of the Central Region and the southeast of Madagascar, with an isolated occurrence on the west coast (Fig. 64). Mt5 is less common and shows a more patchy distribution (Fig. 65) than the other four morphotypes; it is sympatric with $\mathrm{mt} 4$ at some and with $\mathrm{mt} 1$ and $\mathrm{mt} 2$ at only a few localities.

Within its distribution range, the $C$. hova-complex is the most abundant and dominant representative of the Malagasy Decacrema. Although these ants possess an arboreal lifestyle, they are nonetheless highly conspicuous on the forest floor when foraging for food and nesting material, and could hardly be missed in an inventory. Fisher et al. collected workers of these ants innumerable times from leaf litter and beating samples, as well as pitfall, yellow pan and malaise traps. Prevailing nesting behaviour of the C. hova-complex however is the construction of arboreal carton nests. I have found these nests in various heights in the canopy: from as low as $1.20 \mathrm{~m}$, attached to vines or branches of young trees, to $20 \mathrm{~m}$ or more high up in the crowns of canopy- 
level trees. The sizes of carton nests inhabited by workers, the reproductive queen and brood range from as small as $6 \times 4 \mathrm{~cm}$ in diameter to as large as $25 \times 20 \mathrm{~cm}$. Nests housing only workers are frequently encountered and can be smaller in size. It is unclear whether these queenless colonies represent satellite nests of larger nests that are hard to locate, or aggregations of workers whose colony underwent disturbances that resulted in the loss of their reproductive queen and brood. Polydomy in these carton-nesting colonies seems to be quite common, and two or more nests often can be found very close to each other on the same tree, or located on adjacent trees. Experimental or genetic studies would be needed to fully explore details of colony structure. In 17 carton nests out of a total of 30 dissected nests I found one dealate queen together with workers and brood, sometimes with alate queens and males as well. The remaining 13 nests contained only workers, sometimes together with brood. Carton nest colonies further housed staphylinid beetles ( 6 colonies), and in one case scale insects of an undetermined genus of the family Monophlebidae, possibly Gigantococcus (P.J. Gullan, pers. comm.).

There are some records in the CASC of C. hova-complex workers (in some cases with dealate queens) collected from dead twigs or branches, and in a single instance I have found workers with brood in a dead twig myself. It seems likely that colony founding within the species-complex takes place in dead twigs or branches and the construction of carton nests subsequently commences after a certain colony size is reached, i.e. when the colony can afford to send workers to forage for nest material rather than food. The ultimate domicile may always be the carton nest, since this can support much larger numbers of individuals and can be extended in size as the colony grows.

Etymology. The C. hova-complex is named for the oldest available species name that belongs under the complex, $C$. hova Forel. The Malagasy word "hova" denotes the inhabitants of the region Imerina on the east coast of Madagascar, where the type locality of $C$. hova lies.

\section{Crematogaster mahery NEW SPECIES}

(Fig. 35 \& 41)

Holotype worker. MADAGASCAR: R.S. Manombo, -23.01580, 47.71900, 30m, 21-22.iv.2006, ex dead twig above ground, littoral rainforest, B.L.Fisher et al. \#14050 (CASENT0071537, specimen image on antweb) (deposited in CASC).

Paratypes. 2w, R.S. Manombo, -23.01580, 47.71900, 30m, 21-22.iv.2006, ex dead branch above ground, littoral rainforest (B.L.Fisher et al. \#14055: CASENT0193587 and CASENT0193588); 2w, P.N. Andringitra, -22.22350, 47.01176, 780m, 8-9.iii.2010, ex carton nest, riparian rainforest edge (B.B.Blaimer \#228: CASENT0193558 and CASENT0193549) (deposited in MCZC, SAMC, MHNG, UCDC).

Material examined. (BBBC, CASC) MADAGASCAR Toamasina: P.N. Zahamena: -17.75244, 48.85320, 760m; -17.75908, 48.85468, 780m; -17.74300, 48.72936, 860m , -17.73360, 48.72625, 950m (B.L.Fisher et al.); Fianarantsoa: Rés. Andringitra [=P.N. Andringitra]: -22.23333, 47.00000, 825m (B.L.Fisher); P.N. Andringitra: -22.22350, 47.01176, 780m (B.B.Blaimer); R.S. Manombo: -23.01580, 47.71900, 30m (B.L.Fisher et al.); Toliara: P.N. Andohahela: -24.76389, 46.75167, 900m (B.L.Fisher et al.); 13km NW Enakara, Rés. Andohahela [= P.N. Andohahela]: -24.55000, 46.80000, 1250m (B.L.Fisher).

Worker measurements (n=11). HW 1.09-1.21; HL 0.98-1.12; EL 0.24-0.28; SL 0.87-0.97; WL 1.181.32; SPL 0.23-0.41; PTH 0.22-0.26; PTL 0.32-0.38, PTW 0.30-0.39, PPL 0.21-0.28, PPW 0.28-0.37; LHT 0.99-1.07; CI 1.06-1.20; OI 0.23-0.29; SI 0.83-0.92, SPI 0.20-0.33; PTHI 0.64-0.76; PTWI 0.87-1.11; PPI 1.26-1.62; LBI 1.15-1.23.

Worker description. Large to very large (HW 1.09-1.21, WL 1.18-1.32).

Masticatory margin of mandibles with 5 teeth; posterior margin of head straight, laterally subangular; antennal scapes surpassing posterior margin of head easily; midline of eyes situated at or above midline of head in full face view.

Pronotum laterally angular; promesonotal suture broadly impressed, usually complete and continuing laterally to mesopleural spiracle, thereby distinctly constricting mesonotum in relation to pronotum; outline of 
promesonotum more or less flat in lateral view; antero-medial portion of mesonotum from slightly to prominently raised in respect to pronotum, medially tuberculate; dorsal face of mesonotum medially flat, lateral portion broadly angular; mesonotum postero-laterally angular or tuberculate, and with a distinct posterior face; propodeal spines massive, medium to large (SPI 0.20-0.33), at least longer than width between their bases, evenly tapering, in lateral view from straight to strongly upwards curved, in dorsal view usually moderately to strongly diverging $\left(>20^{\circ}\right)$; petiole in dorsal view usually hexagonal, not lobed, lacking dorsolateral carinae or tubercule; dorsum of petiole flat; subpetiolar process variable, articulated as round or angular antero-ventral tooth or close to absent.

Head sculpture aciculate; mesosoma with mesopleuron partly shiny, partly rugulose, and metapleuron carinulate to costulate; dorsal face of propodeum costulate, posterior face largely shiny; otherwise mesosoma including legs largely aciculate; dorsal face of petiole mostly shiny, laterally and ventrally coarsely reticulate; postpetiole dorsally feebly rugulose, ventrally reticulate; helcium carinulate; face with 4-6 erect setae; promesonotum with 2 erect humeral setae and sometimes 2 erect setae laterally present on mesonotum; petiole and postpetiole lacking erect pilosity.

Colour medium brown to black, if black then abdominal segment 4-7 sometimes of brown or ochre coloration.

Variation. Populations from P.N. Zahamena and R.S. Manombo possess strongly upwards curved and slightly shorter spines, whereas the P.N. Andringitra and P.N. Andohahela populations have straight, but therefore longer spines.

Comments. This species is closely related to the $C$. hova-complex and C. sabatra and is hardly separable from the former in the field. It may further be confused with both even under the microscope. Workers of the $C$. hova-complex reaching the body size of $C$. mahery usually possess a costulate or areolate head sculpture, not strictly aciculate as in $C$. mahery. Furthermore, although in size and other characters very similar to $C$. sabatra, there is only opportunity for confusion of the two at one locality, P.N. Andringitra. Here the two species can be separated by head sculpture: at this locality $C$. sabatra exhibits a reticulate sculpture pattern that can easily be distinguished from the aciculate head sculpture of $C$. mahery. The description of $C$. mahery as a new species is also supported by both mitochondrial and nuclear DNA data.

Distribution and biology. At the moment $C$. mahery is known only from four localities in Madagascar (Fig. 55). Three localities (P.N. Zahamena, Andringitra and Andohahela) harbour mid-elevation to montane rainforest habitats, the fourth (R.S. Manombo) is home to a rare patch of littoral forest on the east coast. These forests are situated roughly between $250 \mathrm{~km}$ and $450 \mathrm{~km}$ away from each other along a trajectory of $700 \mathrm{~km}$ across the island. It is an intriguing question how this disjunct distribution came about, since it appears that similar potential habitat exists between those localities. A further striking contrast is the species' distribution in mountainous regions at higher elevations on the one hand, and in the littoral forest at sea level on the other hand. This is the sole Decacrema species recorded from the littoral forest of the R.S. Manombo.

C. mahery co-occurs with the hova-complex at three localities (P.N. Zahamena, Andringitra and Andohahela), and at one locality (P.N. Andringitra) it is also sympatric with C. sabatra and C. malala. Collections mostly comprise workers from beating samples and pitfall and malaise traps, and queens or males of $C$. mahery have not yet been collected. At R.S. Manombo, Fisher et al. collected workers nesting in dead twigs and branches and in rotten logs. In the Andringitra massif, I was fortunate to find a single colony of these ants nesting in three separate carton nests in a small, solitary tree in an open, riverine habitat. Dissection of the nests yielded only workers and brood, but no queen. This colony housed pseudococcids of an undescribed species of the genus Tylococcus (P.J. Gullan, pers. comm.), feeding inside the carton structure on the main branch around which the nest was constructed.

Etymology. "Mahery" means "powerful" in Malagasy, refering to the large body size of workers of this species. The word "mahery" is used in this combination as a noun in apposition. 


\section{Crematogaster malala NEW SPECIES}

(Fig. $36 \& 42$ )

Holotype worker. MADAGASCAR: P.C. Ankazomivady, -20.79751, 47.17910, 1782m, ex dead twig above ground, disturbed montane rainforest, B.L.Fisher et al. \#20466 (CASENT0140925, specimen image on Antweb) (deposited in CASC).

Paratypes. 4w, P.N. Befotaka-Midongy, -23.84080, 46.95750, 1250m, ex dead twig above ground, montane rainforest, (B.L.Fisher et al. \#15029: CASENT0193561 and CASENT0193562; \#15041: CASENT0193559 and CASENT0193560) (deposited in MCZC, SAMC, MHNG, UCDC).

Material examined. (CASC) MADAGASCAR Fianarantsoa 36km S Ambalavao, Rés. Andringitra (=P.N. Andringitra): -22.23333, 47.00000, 1925m (B.L.Fisher); P.N. Andringitra: -22.18767, 46.90083, 2150m (B.L.Fisher et al.); P.N. Befotaka-Midongy: -23.84080, 46.95750, 1250m (B.L.Fisher et al.); 28km SSW Ambositra, Ankazomivady [=P.C. Ankazomivady]: -20.77500, 47.16833, 1680m (B.L.Fisher et al.); P.C. Ankazomivady: -20.77667, 47.16500, 1780m; -20.79751, 47.17910, 1782m (B.L.Fisher et al.); R.S. Ivohibe: $-22.49667,46.95500,1575 \mathrm{~m}$ (B.L.Fisher et al.).

Worker measurements (n=10). HW 0.89-1.09; HL 0.80-0.99; EL 0.21-0.25; SL 0.73-0.82; WL 0.971.16; SPL 0.18-0.27; PTH 0.20-0.24; PTL 0.23-0.28; PTW 0.29-0.41; PPL 0.17-0.21; PPW 0.26-0.36; LHT 0.70-0.88; CI 1.05-1.17; OI 0.24-0.28; SI 0.81-0.91; SPI 0.17-0.25, PTHI 0.84-1.00, PTWI 1.18-1.64, PPI 1.43-1.72; LBI 1.23-1.39.

Worker description. Small to medium species (HW 0.89-1.09, WL 0.97-1.16).

Masticatory margin of mandibles with 4 teeth; posterior margin of head laterally forming round corners; antennae with scapes reaching or just surpassing posterior margin; midline of eyes situated just above midline of head in full face view.

Promesonotum with laterally rounded shoulders; promesonotal suture weakly impressed, usually complete; outline of promesonotum convex in lateral view, sometimes broken by mesonotum which is often slightly raised over pronotum and forming a small antero-medial tubercle; mesonotum postero-laterally rounded or angular; propodeal spines medium sized (SPI 0.17-0.25), less than half the width between their bases, evenly tapering, from straight to weakly curved, and in dorsal view weakly diverging; petiole in dorsal view trapezoidal, anteriorly flared into rounded lobes, dorso-lateral margins carinate, terminating in a small postero-lateral tubercle; subpetiolar process present as a long, moderately acute antero-ventral tooth; postpetiole distinctly bilobed and broadly medially impressed.

Head sculpture reduced, usually aciculate; mesosoma with meso-and metapleuron carinulate or costulate; dorsal face of propodeum weakly costulate to reticulate; posterior face largely shiny; rest of mesosoma including legs largely aciculate; dorsal face of petiole mostly shiny; postpetiole dorsally feebly reticulate; lateral and ventral face of petiole and postpetiole coarsely rugulose-reticulate; helcium dorsally carinulate; face with 2-4 erect setae; pilosity on dorsum of mesosoma 2-4 erect humeral setae, rarely one pair of erect setae at base of mesonotal tubercules; a pair of stiff, long, erect setae present on postero-lateral tubercles of petiole; dorso-posterior erect setae also present on postpetiole; abdominal tergites 4-7 with scattered erect pilosity.

Colour light to dark brown, mesosoma usually lighter coloured than head and metasoma.

Variation. Workers from the Midongy region are darker coloured than from P.C. Ankazomivady and P.N. Andringitra localities and have a denser appressed pubescence. The mesonotum is often more prominently raised over the pronotum in the P.C. Ankazomivady and P.N. Midongy material, than in workers from the other locations.

Comments. Crematogaster malala is characterized by a combination of the following features. The petiole has postero-lateral tubercles that each bear a single, stiff and long erect seta. The postpetiole is distinctly bilobed and broadly medially impressed and also bears a pair of dorso-posterior stiff, long erect setae. These characters, together with the erect pilosity on abdominal tergites 4-7 and the 4 mandibular teeth, are shared with $C$. grevei. In distinction to $C$. grevei however the lateral portions of the promesonotum in $C$. malala are not raised with respect to the median portion and are postero-lateral rounded or angular. The 
metanotal groove further lacks lateral carinae and the promesonotum forms a convex outline in lateral view. In molecular phylogenetic analyses this species is well supported as a distinct clade by both nuclear and mitochondrial data, and results suggest it is the sister taxon to the rest of the Malagasy Decacrema species.

Distribution and biology. Collections of this species are rare, with a few colonies from three locations of higher elevation rainforests $(\geq 1250 \mathrm{~m})$ in the Ankaratra (Ankazomivady), Andringitra and Midongy massifs (Fig. 56). The isolated, montane distribution invites speculation that glacial cycles have played an important role in shaping this species' evolutionary history and distribution, as has been suggested for other taxa in Madagascar (for an overview see Vences et al., 2009). Next to nothing is known about the biology of this species. Fisher's collections of $C$. malala workers document these ants as nesting in dead twigs $(n=4)$ and in carton nests $(\mathrm{n}=1)$; queens or males of this species have not yet been discovered. A recent search for $C$. malala in the P.N. Andringitra at about $1800 \mathrm{~m}$, not far from where it had been collected before, was unsuccessful and suggests that these ants might be highly restricted in habitat and possess inconspicuous life habits.

Etymology. This species' name is derived from the Malagasy adjective "malala", meaning "the beloved one", inspired by happiness about the discovery of its morphological distinctness compared to the other species in the group.

\section{Crematogaster nosibeensis Forel 1891 NEW STATUS}

(Fig. 32 \& 38)

Crematogaster hova $\mathrm{r}$. nosibeensis Forel,1891: 180. Queen syntypes from MADAGASCAR, Nosibé (O'swald) [Hamburg Museum] [not examined, destroyed in WWII].

Crematogaster nosibeensis Forel, Dalla Torre, 1893: 84. Raised to species.

Crematogaster hova nosibeensis Forel, Wheeler, 1922b: 1026. Combination in C. (Decacrema); subspecies of hova.

Crematogaster hova nosibeensis Forel, Emery, 1922: 138. Subspecies of hova.

Neotype queen. MADAGASCAR: Nosy Bé, R.N.I. Lokobé: -13.41944, 48.33117, 30m, 19.-24.iii.2001, at light, rainforest, B.L. Fisher et al. \#3420 (CASENT0436030; specimen image on Antweb) (deposited in CASC).

Material examined. (BBBC, CASC, MHNG, PSWC) MADAGASCAR Antsiranana: Ampasindava, Ambilanivy: -13.79861, 48.16167, 600m (B. L. Fisher et al.); Nosy Bé, R.N.I. Lokobé: -13.41944, 48.33117, 30m (B.L.Fisher et al.) and -13.41670, 46.30000, 0-400m (D.M.Olson); Nosibé [Nosy Bé] (Völtzkow); R.S. Manongarivo: -13.96167, 48.43333, 400m; -13.97667, 48.42333, 780m (B.L.Fisher et al.); R.S. Manongarivo: -13.92950, 48.45320, 275m (B.B.Blaimer); R.S. Ambre: -12.46889, 49.24217, 325m (B.L.Fisher et al.); Antananarivo: R.S. Ambohitantely: -18.22528, 47.28683, 1410m, -18.17140, 47.28182, 1620m (Rabeson et al); R.S. Ambohitantely: -18.21722, 47.28470, 1550m; -18.17272, 47.28023, 1610m (B.B.Blaimer).

Worker measurements $(\mathrm{n}=13)$. HW $0.86-1.04$; HL $0.75-0.94$; EL $0.19-0.24$; SL $0.64-0.81$; WL 0.80 1.05; SPL 0.13-0.21; PTH 0.17-0.22; PTL 0.24-0.30; PTW 0.26-0.37; PPL 0.17-0.22; PPW 0.23-0.31; LHT 0.65-0.88; CI 1.12-1.18; OI 0.23-0.27; SI 0.81-0.90; SPI 0.15-0.20; PTHI 0.69-0.79; PTWI 1.07-1.37; PPI 1.26-1.53; LBI 1.17-1.28.

Worker description. Small to medium size (HW 0.86-1.04, WL 0.80-1.05).

Masticatory margin of mandibles with 5 teeth; posterior margin of head straight, laterally forming round corners; antennae with scapes easily surpassing posterior margin; midline of eyes situated at midline of head in full face view.

Pronotum laterally subangular; promesonotal suture varying from completely impressed to absent; outline of promesonotum flat in lateral view; antero-medial portion of mesonotum either slightly raised over pronotum (but never tuberculate), or indistinguishable from pronotum; dorsal face of mesonotum flat, lateral portions broadly angular; mesonotum postero-laterally angular and usually with a distinct posterior face; dorsal face of propodeum absent to very short; propodeal spines small-medium sized (SPI 0.15-0.20), between half and full the width between their bases, thin and needle-shaped, slowly tapering, straight or 
down-curved, in dorsal view weakly diverging $\left(<20^{\circ}\right)$; propodeal spiracle large, much larger than base of spines; petiole in dorsal view weakly to distinctly lobed; subpetiolar process articulated as broad, rounded protuberance.

Head sculpture aciculate; promesonotum dorsally aciculate with shiny parts; mesopleuron largely shiny, metapleuron carinulate to reticulate; posterior face of propodeum largely shiny; petiole dorsally mostly shiny, laterally and ventrally coarsely rugulose-reticulate; postpetiole dorsally rugulose, ventrally rugulosereticulate; helcium carinulate; erect pilosity on face reduced to 2 setae at base of frontal carinae; ventral pilosity on head reduced, ventral suberect pilosity absent; pilosity on dorsum of mesosoma usually confined to 2 erect humeral setae; petiole lacking erect pilosity; postpetiole with or without short postero-lateral setae.

Two variants. Either colour brown to black, with mesosoma usually lighter coloured than head and metasoma (R.N.I. Lokobé, R.S. Manongarivo, Ampasindava, R.S. Ambre; as shown in Fig 32 \& 38), or distinctly bi-coloured with head, mesosoma and part of A4 reddish to orange and rest of metasoma dark brown (R.S. Ambohitantely; specimen image on Antweb: CASENT0423447).

Queen measurements (n=3). HW 1.64-1.72, HL 1.37-1.39, EL 0.43-0.45, SL 0.92-0.94, MSNW 1.331.37, MSNL 2.06-2.07, PTH 0.34-0.38, PTL 0.41-0.47, PTW 0.44-0.54, PPL 0.39-0.43, PPW 0.47-0.54, SPL 0.12-0.16, WL 2.20-2.29, LHT 1.06-1.10, CI 1.20-1.24, OI 0.31-0.33, SI 0.66-0.68, MSNI 0.64-0.66, PTHI 0.71-0.93, PTWI 0.95-1.33, PPI 1.18-1.26, SPI 0.05-0.07, LBI 2.08-2.09.

Queen description. Medium size (HW 1.64-1.72; WL 2.20-2.29). With worker characters except as described below.

Antennal scapes not surpassing posterior margin of head; midline of eyes situated below midline of head in full face view; posterior margin straight.

Mesosoma short and compact (MSNI 0.64-0.66, WL 2.20-2.29); mesoscutum in dorsal view subcircular, short, about as long as wide; scutellum in lateral view greatly projecting over postscutellum; mesopleuron with episternal groove weakly impressed; in lateral view mesepisternum meeting pronotum in nearly perpendicular angle; dorsal face of propodeum very short; propodeal suture shallow, but laterally reaching level of propodeal spiracle; propodeal spines ranging from denticles to very sharp points, never reduced to tubercules; petiole somewhat flattened anteriorly in lateral view; dorsal face of petiole flat; subpetiolar process reduced to small angular antero-ventral tooth; metasoma oval-shaped in dorsal view, shorter and broader than in other Decacrema species; abdominal segment 4 narrowed anteriorly.

Propodeum with a single transverse ridge or carinae marking the border to posterior face; petiole reticulate throughout; postpetiole feebly reticulate throughout; 10-12 erect setae on face; mesonotum with 20-24 erect setae, but leaving median portion of mesonotum devoid of erect pilosity; dorso-posterior short, erect setae on petiole and postpetiole present, in addition petiole and postpetiole with lateral suberect to erect setae and scattered appressed pubescence.

Meso- and metasoma brown, head reddish brown, legs brown to dark brown, metasoma brown to dark brown.

Variation. This species displays interesting bimodal variation in coloration, similar to that expressed in $C$. grevei. Workers of populations from northern Madagascar (R.N.I. Lokobé, R.S. Manongarivo, Ampasindava, R.S. Ambre) are brown or black, whereas the isolated population from the central highlands of Madagascar (R.S. Ambohitantely) is bi-coloured red-orange and black. Individuals from the latter are also generally larger. Queens lack the variation in colour.

Comments. Elevation from subspecies to species rank for $C$. nosibeensis is warranted beyond morphology by analyses of nuclear DNA data that place this species firmly outside the $C$. hova-complex. The sympatric occurrence of $C$. nosibeensis with the $C$. hova-complex at all localities, except R.N.I. Lokobé, further supports this decision. The conspecificity of the northern populations with the R.S. Ambohitantely population is established by both morphology and nuclear DNA data. The designation of a neotype for the species is justified for the following reasons.

1) A name-bearing type is necessary to support the rank elevation of $C$. nosibeensis and to clearly demarcate this species from the C. hova-complex. Both queens and workers of this species are easily distinguished from the $C$. hova-complex by a combination of characters. Diagnostic for queens is the short 
and compact mesosoma, the short propodeum with a transverse ridge or carinae bordering the propodeal declivity, and the acute propodeal spines compared to queens in the C. hova-complex. Workers are recognizable by means of their blunt or broadly rounded subpetiolar process, the postero-lateral angular promesonotum, and their reduced sculpture.

2) Confirmation has been obtained from the "Zoologisches Museum" in Hamburg, Germany that the queen syntypes of $C$. hova nosibeensis collected by M. O'swald were among the collections of this museum destroyed in World War II (R. Peters, pers comm.).

3) The conspecificity of the neotype with the former name-bearing types seems secured since it agrees perfectly with the original description. Moreover, this is the only species in the Decacrema-group that occurs at the type locality, the island of Nosy Bé [= Nosibé, the old spelling in the original description (United States Board on Geographical Names, 1989)], from where the collections of M. O'swald are documented (Forel, 1891).

A queen collected from the type locality Nosy Bé is hereby chosen as the neotype and a full description is presented above. For the benefit of having a comprehensive type collection in one place, the neotype is deposited together with the other type material in the CASC, not in Hamburg.

Distribution and biology. This species shows a distribution mostly restricted to low and mid-elevation rainforests of the Sambirano Region of Madagascar's northwest, including the island of Nosy Bé, the Ampasindava peninsula and the Manongarivo massif (Fig. 57). The Sambirano forests are remarkable in plant composition, harboring high local endemism (Gautier \& Goodman, 2003). Isolated populations of Crematogaster nosibeensis exist in the transitional dry forests of the R.S. Ambre in the foothills of the Montagne d'Ambre massif in the far north of Madagascar and the R.S. Ambohitantely in the central highlands. Ambohitantely forest is one of the last remnants of montane transition forest in this region as most of the surrounding vegetation has been altered into grasslands, probably due to excessive burning related to agricultural practices. It seems therefore likely that the species once spanned a more continuous distribution reaching from Madagascar's north southwards to the central highlands.

In the R.S. Manongarivo, I collected workers of C. nosibeensis from a trunk of Calantica (Salicaceae; vernacular name "Janganito") that were transporting wood fibers to a carton nest out of reach for any of my collection methods. I therefore assume that this species makes carton nests in the Manongarivo region, although it has never been collected from a carton nest at any of the northern localities. Fisher et al. collected workers of $C$. nosibeensis with various ground-sampling techniques, but never in association with its nest site. In R.S. Manongarivo this species certainly belongs to the rarer and less dominant species of Crematogaster, since I was able to make no more than two collections during a period of four collecting days.

In striking contrast however, $C$. nosibeensis is a conspicuous and dominant element of the canopy ant fauna in R.S. Ambohitantely, side by side with the C. hova-complex. Here I found the species nesting exclusively in carton nests, mostly constructed high up (ca. 15-25m) in the canopy. One remarkable collection event involved two very large, polydomous carton nest colonies that had nests in neighbouring trees of the endemic genus Sarcolaena (Sarcolaenaceae). Each colony consisted of two large $(15 \times 15 \mathrm{~cm}-20 \times 25 \mathrm{~cm})$ nests housing either workers, reproductives and brood, or just workers and brood. Close to the main nests, 4-6 smaller (ca. $2 \times 1 \mathrm{~cm}-5 \times 5 \mathrm{~cm}$ ) satellite carton nests or shelters were present, containing worker ants and mealybugs of an undescribed species in the genus Tylococcus (Pseudococcidae), which were morphologically close to but distinct from those collected with C. mahery in P.N. Andringitra (P.J. Gullan, pers. comm.). The sole function of these satellite carton shelters appears to be the protection of the mealybugs. Besides workers, a dealate queen (each) and brood, both of these colonies also contained massive numbers of alates, both queens and males. This suggests that this was the height of reproduction for this species in the region (month of February). In contrast, none of the colonies of the C. hova-complex collected in Ambohitantely possessed alates - the temporal separation of reproduction may act as an important mechanism of reproductive isolation between these sympatric species. 


\section{Crematogaster sabatra NEW SPECIES}

(Fig. $34 \& 40$ )

Holotype worker. MADAGASCAR: P.N. Befotaka-Midongy, -23.84080, 46.95750, 1250m, 17.-19.xi.2006, beating low vegetation, montane rainforest, B.L.Fisher et al. \#15075 (CASENT0193162, specimen image on antweb) (deposited in CASC).

Paratypes. 4w, same locality as holotype, ex dead branch above ground (B.L.Fisher \#15066, CASENT0193541, CASENT0193554, CASENT0193553 and CASENT0193552) (deposited in MCZC, SAMC, MHNG, UCDC).

Material examined. (CASC) MADAGASCAR: Fianarantsoa: 29km SSW Ambositra, Ankazomivady [=P.C. Ankazomivady]: -20.77667, 47.16500, 1700m (B.L.Fisher); 40km S Ambalavao, Rés. Andringitra (=P.N. Andringitra): -22.21667, 46.96667, 1275m (B.L.Fisher); R.S. Kalambatritra: -23.46350, 46.46310, 1270m (B.L.Fisher et al.); P.N. Befotaka-Midongy: -23.84080, 46.95750, 1250m (B.L.Fisher et al.).

Worker measurements $(\mathrm{n}=10)$. HW 1.15-1.31; HL 1.03-1.15; EL 0.26-0.30; SL 0.86-0.98; WL 1.201.34; SPL 0.27-0.42; PTH 0.22-0.25; PTL 0.29-0.38; PTW 0.32-0.37; PPL 0.20-0.23; PPW 0.28-0.31; LHT 0.96-1.10; CI 1.09-1.15; OI 0.23-0.28; SI 0.84-0.88; SPI 0.22-0.33; PTHI 0.60-0.84; PTWI 0.89-1.26; PPI $1.31-1.52$; LBI $1.16-1.24$

Worker description. Large to very large species (HW 1.15-1.31,WL 1.20-1.34).

Masticatory margin of mandibles with 5 teeth; posterior margin of head straight, laterally forming subangular corners; antennal scapes surpassing posterior margin of head easily; midline of eyes situated well above midline of head in full face view.

Pronotum laterally angular; promesonotal suture usually incomplete, impressed only laterally; dorsum of promesonotum more or less flat in lateral view; antero-medial portion of mesonotum from slightly raised to prominently raised with respect to pronotum, but not tuberculate; dorsal face of mesonotum flat, lateral portion from broadly to sharply angular; mesonotum postero-laterally angular or tuberculate, and with a distinct posterior face; propodeal spines massive, medium to large size (SPI 0.22-0.33), at least longer than width between their bases, evenly tapering, in lateral profile fairly straight, in dorsal view moderately diverging $\left(<30^{\circ}\right)$; petiole in dorsal view hexagonal, not lobed, lacking carinae or tubercule; dorsum of petiole flat; subpetiolar process variable, articulated as broadly rounded or angular antero-ventral tooth, or close to absent.

Head sculpture aciculate to reticulate; mesosoma with mesopleuron partly aciculate, partly reticulate, and metapleuron carinulate to costulate; dorsal face of propodeum costulate; posterior face largely smooth and shiny; otherwise mesosoma largely aciculate, or aciculate-reticulate; dorsal face of petiole mostly shiny; lateral and ventral face coarsely reticulate; postpetiole dorsally feebly, ventrally more strongly rugulose; helcium carinulate; face with 4-6 erect setae; promesonotum with 2 erect humeral setae and sometimes 2 erect setae laterally on mesonotum; petiole and postpetiole devoid of erect pilosity.

Colour mostly black, rarely brown.

Variation. Populations from P.N. Andringitra and P.C. Ankazomivady show a mostly reticulate sculpturing on the head and on parts of the promesonotum, in contrast to the aciculate sculpture of workers collected from the southern localities R.S. Kalambatritra and P.N. Befotaka-Midongy.

Comments. Crematogaster sabatra is very closely related to the $C$. hova-complex, from which it can be distinguished by size, the angular postero-lateral character of the mesonotum and the reduced sculpture. The species is further morphologically similar to C. mahery; characters to separate these two species are given above (see description of $C$. mahery). Both mitochondrial and nuclear DNA data support populations of $C$. sabatra from all localities as being conspecific. However, in phylogenetic analysis specimens from the sampled populations (P.N. Andringitra, P.C. Ankazomivady and P.N. Befotaka-Midongy) form a cluster within the $C$. hova-complex, rendering the latter a paraphyletic grouping. The speciation event between $C$. sabatra and the hova-complex thus may have been very recent and reciprocal monophyly is not yet reached, at least in the markers implemented in this study. Considering the sympatry of $C$. sabatra with the $C$. hovacomplex across its entire distribution, strong reproductive isolating mechanisms must already be in place. 
This, together with the morphological distinctness, is here regarded as sufficient evidence to describe $C$. sabatra as a species.

Distribution and biology. Crematogaster sabatra is known only from a few localities in Madagascar: the P.N. Andringitra, P.C. Ankazomivady, P.N. Befotaka-Midongy and R.S. Kalambatritra (Fig. 58). All of these localities are mountainous regions in the Central Region and harbour mid-elevation to montane rainforest, very much reminiscent of the distributions of $C$. mahery and $C$. malala. Crematogaster sabatra has been collected only from elevations of $1250 \mathrm{~m}$ and above (up to $1700 \mathrm{~m}$ ). I surmise that this may be the result of competitive exclusion by the dominant $C$. hova-complex, which is very common at lower elevations in and around these regions, but far less abundant at higher elevations. Queens or males of this species have never been collected, and little information exists on its ecology. Fisher et al. have collected $C$. sabatra mostly while beating vegetation and during other ground sampling methods. On a few occasions colonies were found in dead branches or twigs and carton nests. It seems likely that it shares a very similar arboreal lifestyle as the rest of the Malagasy Decacrema.

Etymology. "Sabatra" is the Malagasy word for "sword", naming the species after its workers' long and sword-like propodeal spines. In this combination "sabatra" is used as a noun in apposition.

\section{Crematogaster sisa NEW SPECIES}

(Fig. 33 \& 39)

Holotype worker. MADAGASCAR: R.S. Manongarivo, -14.02167, 48.41833, 1580m, 27.x.1998, sifted litter (leaf mold, rotten wood), montane rainforest, B.L.Fisher\#1970(49)-2 (CASENT0193556, specimen image on antweb) (deposited in CASC).

Paratypes. 4w, same locality and collection data as holotype (B.L.Fisher\#1970(49)-2, CASENT0193557, CASENT0193542 and CASENT0193543; \#1972(11)-1, CASENT0193009) (deposited in MCZC, SAMC, MHNG, UCDC).

Material examined. (CASC) MADAGASCAR: Antsiranana: R.S. Manongarivo: -14.02167, 48.41833, 1580m; -14.04667, 48.40167, 1860m (B.L.Fisher).

Worker measurements $(\mathrm{n}=10)$. HW $0.89-1.14$; HL 0.81-1.08; EL 0.21-0.27; SL 0.73-0.84; WL 0.911.12; SPL 0.16-0.24; PTH 0.16-0.23; PTL 0.24-0.31; PTW 0.26-0.35; PPL 0.17-0.22; PPW 0.24-0.33; LHT 0.71-0.90; CI 1.05-1.17; OI 0.22-0.28; SI 0.78-0.90; SPI 0.17-0.21; PTHI 0.65-0.83; PTWI 0.91-1.24; PPI 1.33-1.53; LBI 1.23-1.30.

Worker description. Medium to large species (HW 0.89-1.14, WL 0.91-1.12).

Masticatory margin of mandibles with 5 teeth; posterior margin of head straight or medially depressed, laterally forming gentle round corners; $1-3$ ocelli sometimes present on large workers; antennal scapes just surpassing posterior margin of head; midline of eyes situated at midline of head in full face view.

Pronotum laterally angular; promesonotal suture usually complete, and distinctly and broadly impressed, continuing laterally to mesopleural spiracle and thereby constricting mesonotum in relation to pronotum; dorsum of promesonotum more or less flat in lateral view; antero-medial portion of mesonotum slightly raised with respect to pronotum, but not tuberculate; dorsum of mesonotum flat, lateral portion broadly angular; mesonotum postero-laterally angular or tuberculate, and with a distinct to indistinct posterior face; propodeal spines small to medium sized (SPI 0.17-0.21), half as long to as long as width between their bases, evenly tapering, straight or weakly down-curved, in dorsal view weakly diverging; petiole in dorsal view trapezoidal or suboval, weakly lobed or rounded, laterally weakly carinate, with weak postero-lateral tubercle; dorsum of petiole weakly concave; subpetiolar process articulated as acute and distinct antero-ventral tooth, but rarely may be present as only a minute tooth. 

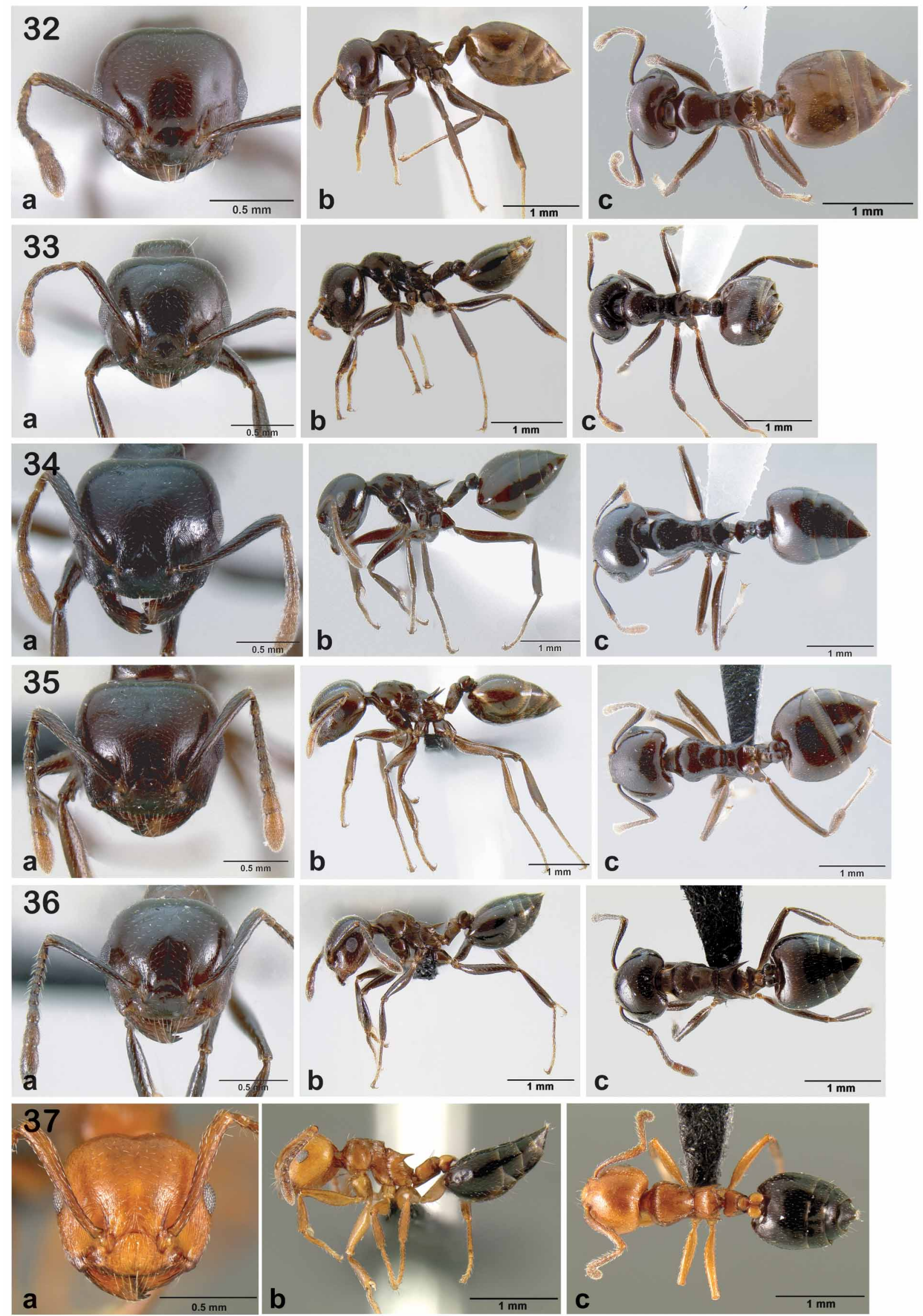

FIGURES 32-37. Malagasy Crematogaster (Decacrema) species; a: full-face view, b: lateral view, c: dorsal view. 32 : C. nosibeensis; 33: C. sisa; 34: C. sabatra; 35: C. mahery; 36: C. malala; 37: C. grevei. 


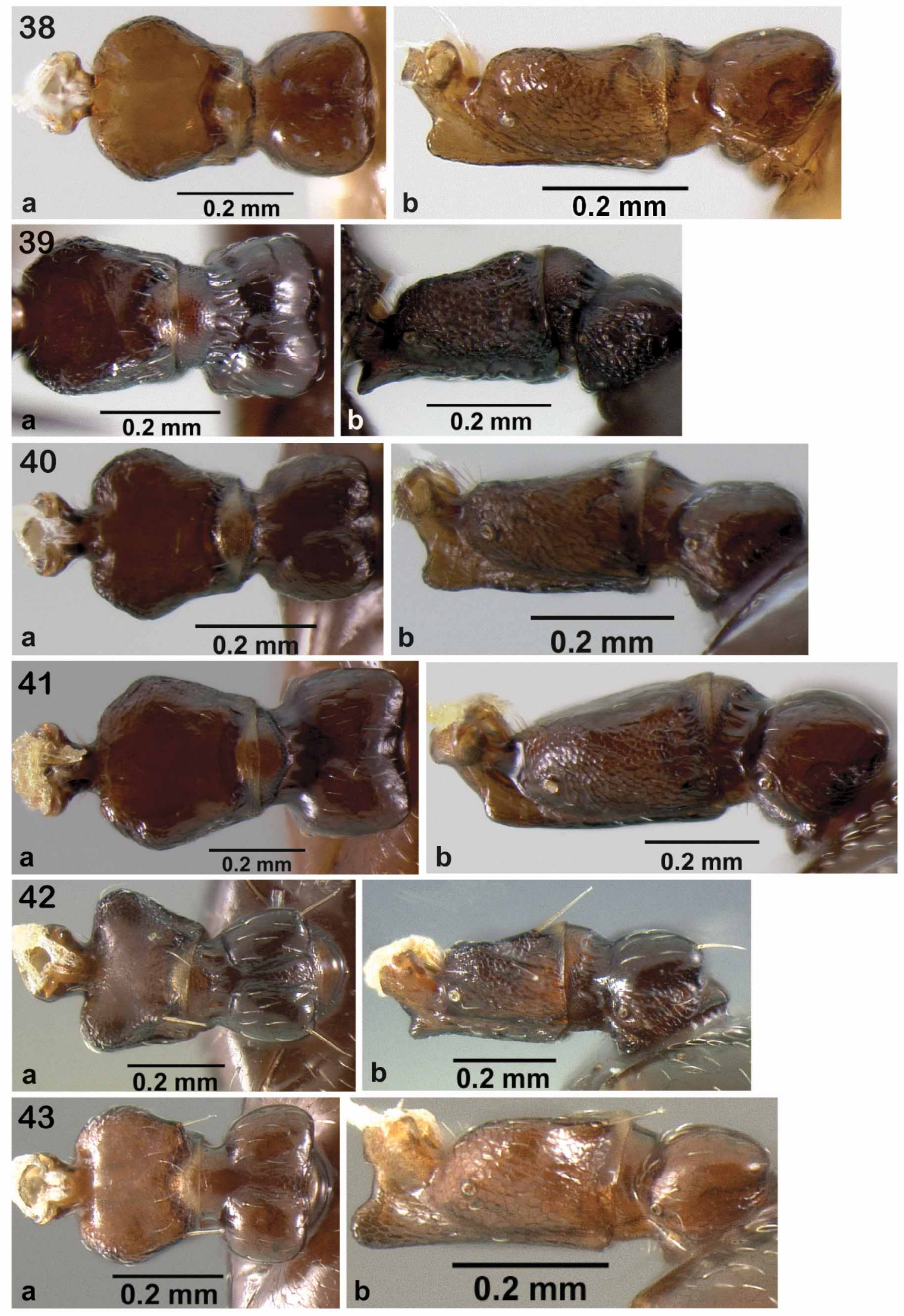

FIGURES 38-43. Malagasy Crematogaster (Decacrema) species; a: petiole \& postpetiole in dorsal view, b: petiole \& postpetiole in lateral view. 38: . nosibeensis; 39: C. sisa, 40: C. sabatra; 41: C. mahery; 42: C. malala; 43: C. grevei. 

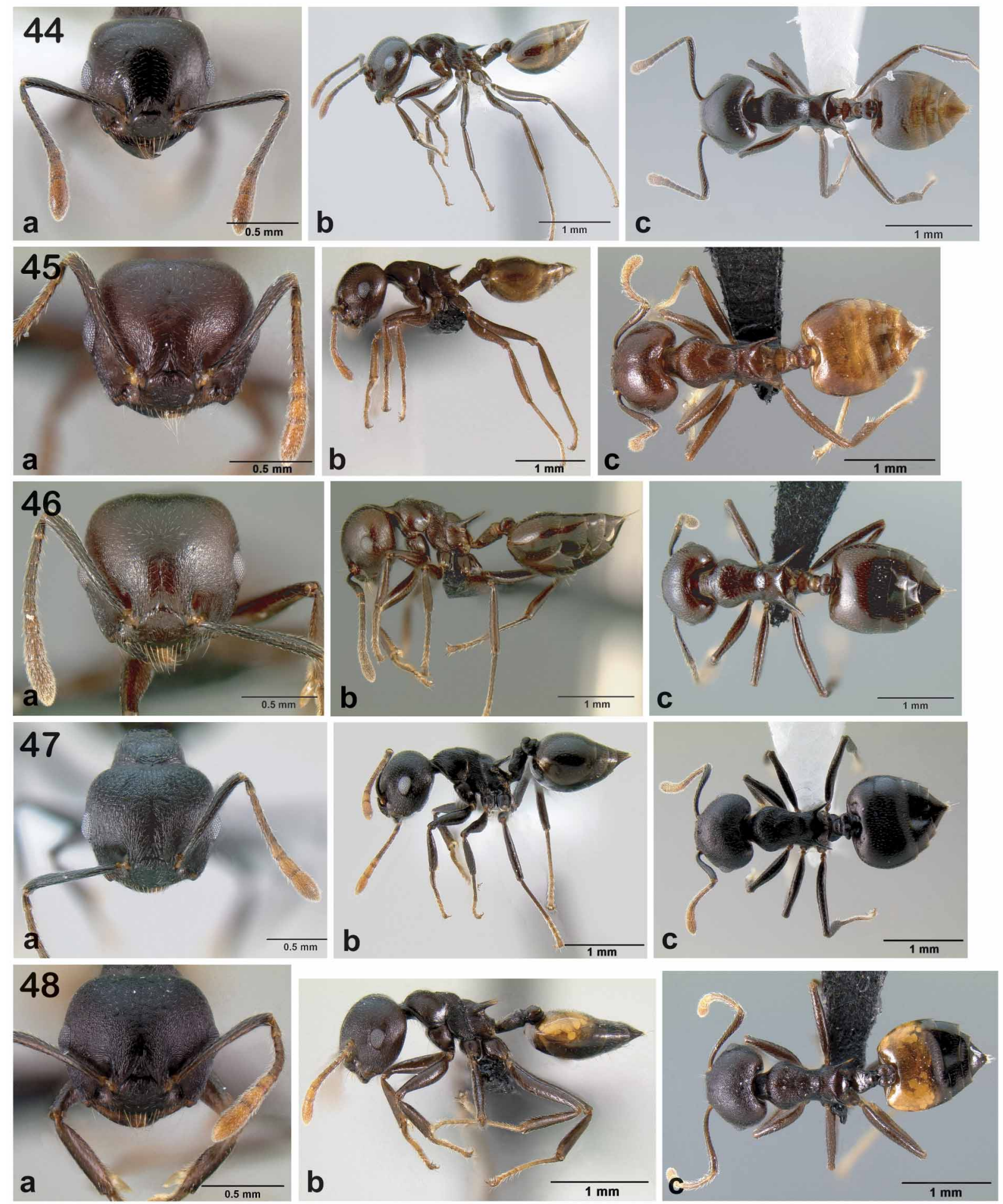

FIGURES 44-48. The Crematogaster (Decacrema) hova-complex; a: full-face view, b: lateral view, c: dorsal view. 44: morphotype 1; 45: morphotype 2; 46: morphotype 3; 47: morphotype 4; 48: morphotype 5.

Head sculpture aciculate; mesosoma with mesopleuron costulate, and metapleuron partly costulate, partly aciculate; dorsal face of propodeum weakly carinulate; posterior face largely shiny; otherwise mesosoma largely aciculate; dorsal face of petiole mostly shiny; lateral and ventral face of petiole coarsely reticulate; postpetiole dorsally and ventrally rugulose; helcium carinulate; face usually with 4-6 erect setae; promesonotum with $0-2$ erect humeral setae; petiole and postpetiole lacking erect pilosity.

Colour brown to dark brown. 


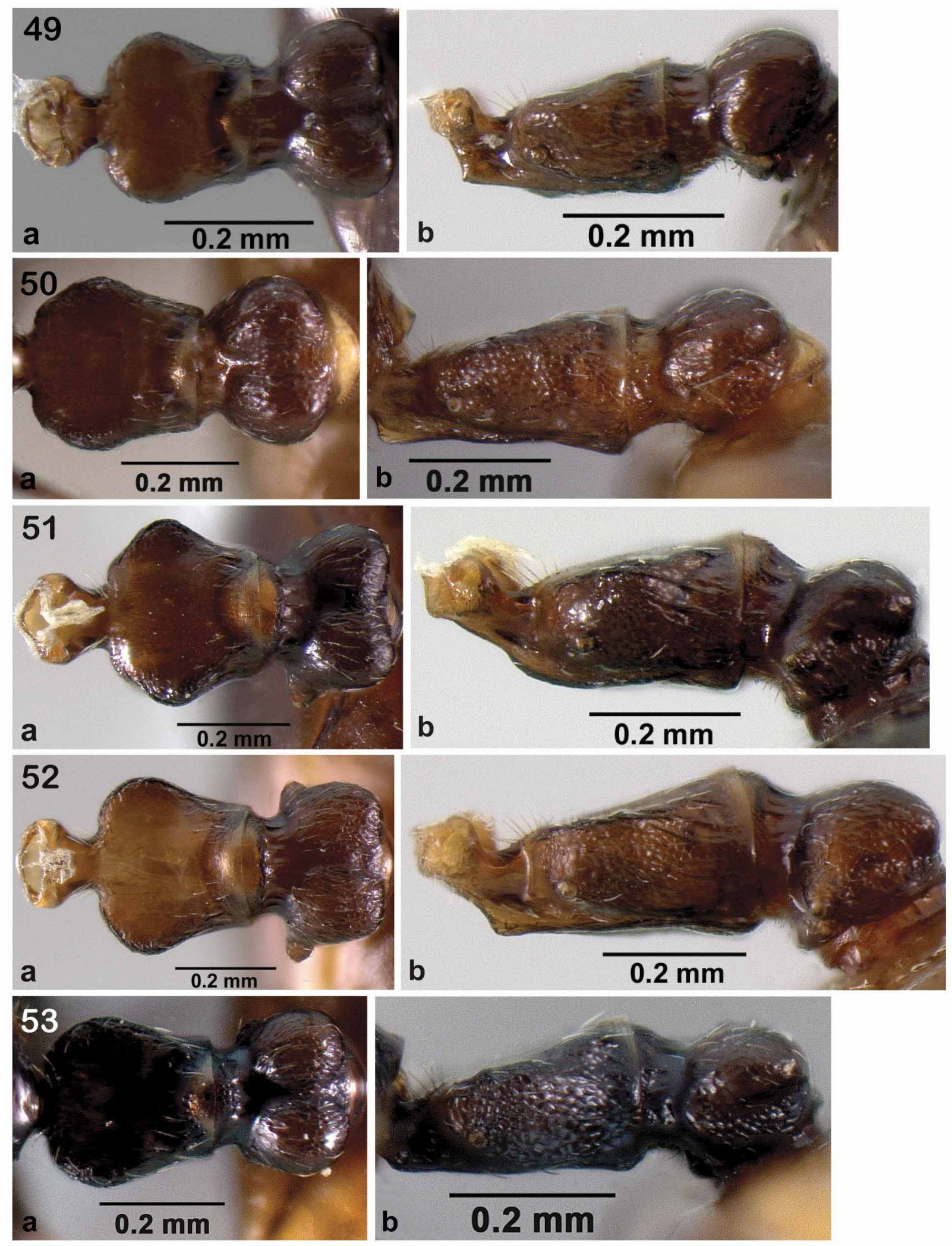

FIGURES 49-53. The Crematogaster (Decacrema) hova-complex; a: petiole \& postpetiole in dorsal view, b: petiole \& postpetiole in lateral view. 49: morphotype 1; 50: morphotype 2; 51: morphotype 3; 52: morphotype 4; 53: morphotype 5. 

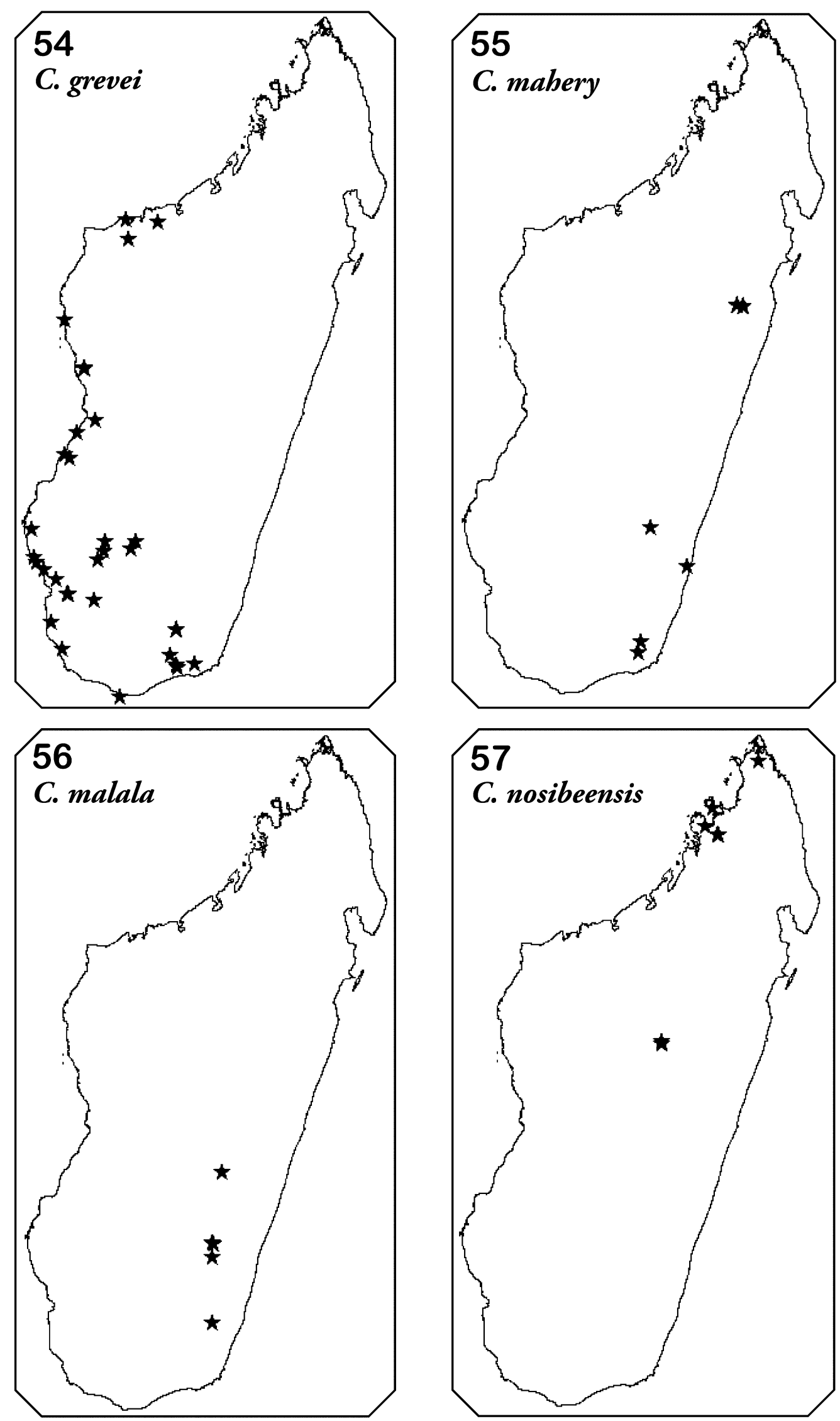

FIGURES 54-57. Distributions of Malagasy Crematogaster (Decacrema). 54: C.grevei; 55: C. mahery; 56: C. malala; 57: C. nosibeensis. 

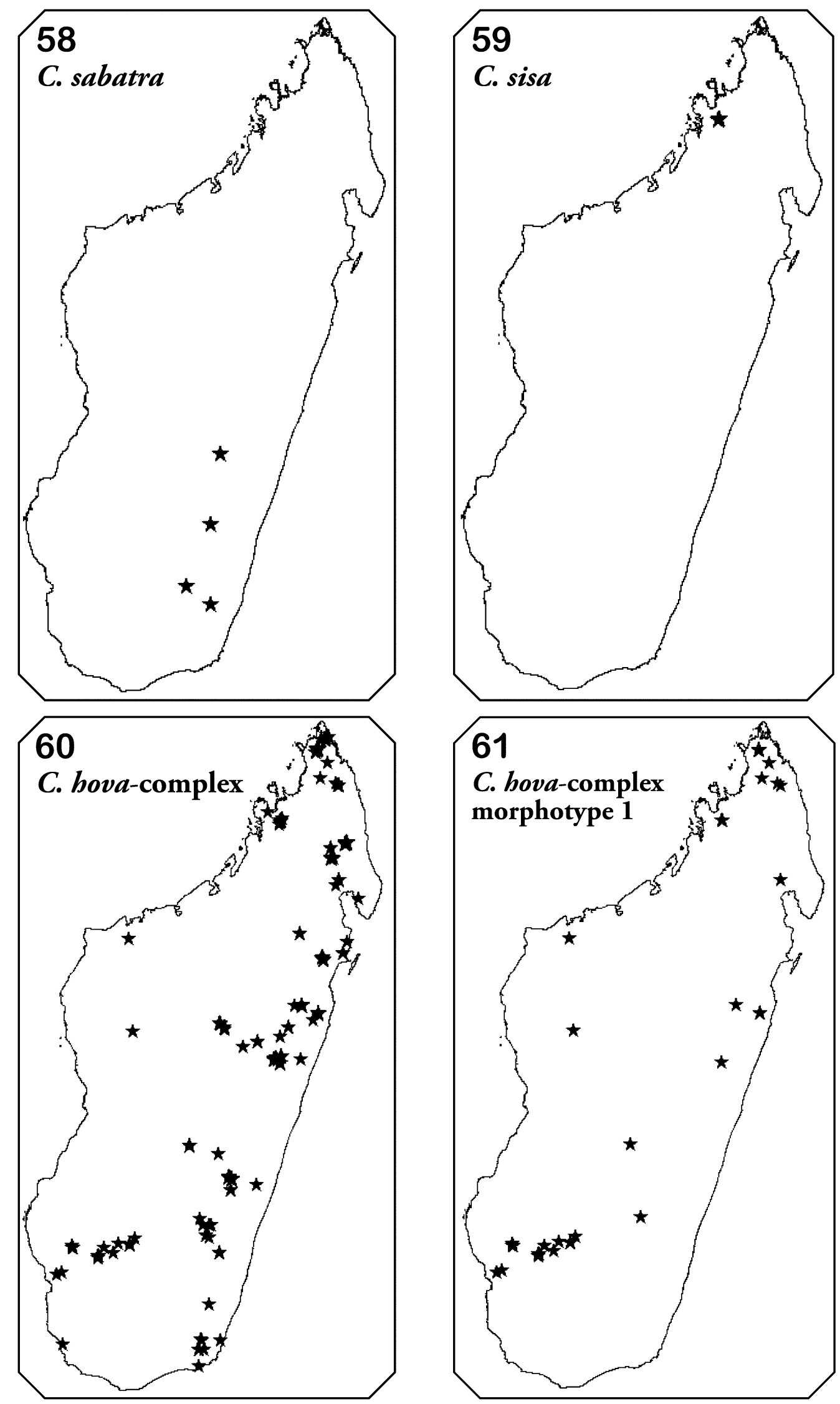

FIGURES 58-61. Distributions of Malagasy Crematogaster (Decacrema). 58: C. sabatra; 59: C. sisa; 60: C. hovacomplex, entire distribution; 61: C. hova-complex morphotype 1. 

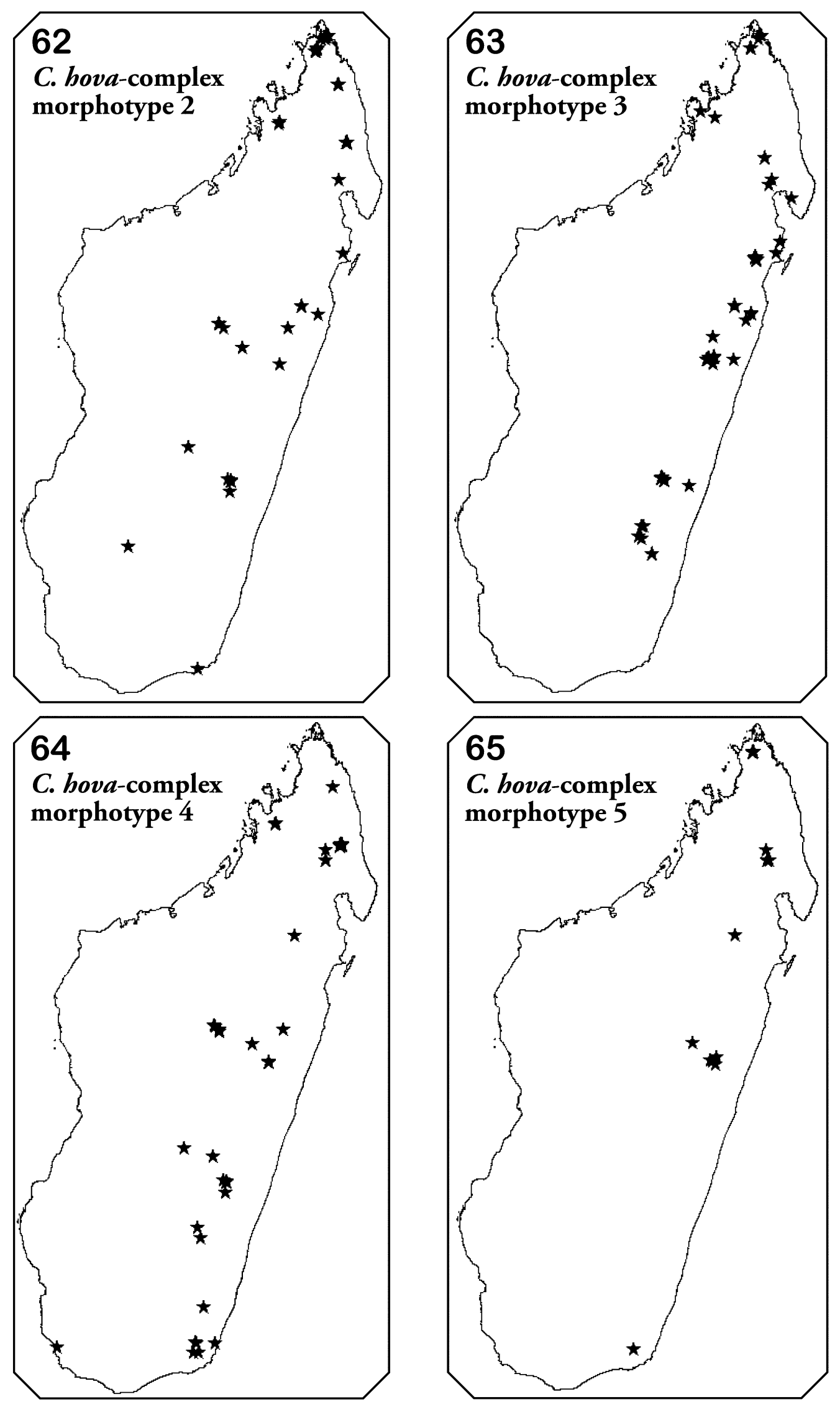

FIGURES 62-65. Distributions of Malagasy Crematogaster (Decacrema). 62: C. hova-complex morphotype 2; 63: $C$. hova-complex morphotype 3; 64: C. hova-complex morphotype 4; 65: Crematogaster hova-complex morphotype 5. 
Variation. There seems to be considerable gradual size variation in workers, but sampling of this species is too sparse to give a general statement.

Comments. This species is easily distinguished morphologically by its uniquely shaped subpetiolar process in form of an acute tooth. The species status of $C$. sis $a$ is further well supported by both mitochondrial and nuclear DNA sequences.

Distribution and biology. Crematogaster sisa has to be regarded as a high-elevation endemic, known to date only from two localities in the rugged and isolated Manongarivo massif (Fig. 59) that were visited during a biological inventory in 1998 by B.L. Fisher (see Fisher, 2002). Collections of foraging workers were made while sifting litter and beating vegetation in montane rainforest habitats at 1580 and $1860 \mathrm{~m}$. No nest collections have yet been recorded for this species, thus reproductives and natural history are entirely unknown. The Manongarivo massif lies in the Sambirano Region of Madagascar's northwest and is part of a mountain complex spanning much of northern Madagascar (Gautier \& Goodman, 2002) - probably one of the least accessible and least studied regions of the island. Further inventory efforts in this region may therefore result in expanding the known distribution of $C$. sisa and lead to the discovery of colonies and their nesting habits. The species occurs in sympatry with the C. hova-complex, but appears to be parapatric in distribution to $C$. nosibeensis by virtue of elevation, since the latter is found in northwest Madagascar only in lowland forests.

Etymology. "Sisa" is Malagasy for "remnant" and this species is named in allusion to its isolated distribution. "Sisa" is used in this combination as a noun in apposition.

\section{Acknowledgments}

For continuous discussions on ideas that led to the publication of this work I am very grateful to P.S.Ward, my fellow lab members at UC Davis and B.L.Fisher. I am further indebted to Brian Fisher for graciously lending me his extensive specimen collection for morphological and molecular research, and whose inventory efforts in Madagascar have made this revisionary work possible. For providing specimens and access to collections, I further thank P.S. Ward, B. Merz, D. Burckhardt, J. Schuberth and F. Koch. Valuable comments on this manuscript that helped to improve this paper were made by P.S. Ward, P.J. Gullan, B.L. Fisher, A. Lucky, J.T. Longino, K. Eguchi and B. Bolton. I further thank Penny Gullan for identifications of Crematogasterassociated mealybugs. Field work in Madagascar would not have been possible without the much appreciated help of T. Razafindrabe, M. Alain, the Malagasy ANGAP personnel, B. Rajemison and B.L. Fisher. Support for this research was provided by the Entomology Department at UC Davis, the Jastro Shields Research Award, the Center for Population Biology at UC Davis, the Systematics Research Fund, the Lewis and Clark Fund for Exploration and Field Research and the Ant AToL NSF grant (EF-0431330, awarded to P.S. Ward).

\section{References}

Arnold, G. (1920) A monograph of the Formicidae of South Africa. (Myrmicinae). Annals of the South African Museum, $14,403-578$.

Bolton, B. (1995) A new general catalogue of the ants of the world. Cambridge, Massachusetts: Harvard University Press, $504 \mathrm{pp}$.

Bolton, B. (2003) Synopsis and classification of Formicidae. Memoirs of the American Entomological Institute, 71, 1370.

Bolton, B., Alpert, G., Ward, P.S. \& Nasrecki, P. (2007) Bolton's catalogue of the ants of the world. In. Harvard University Press.

Brady, S.G., Schultz, T.R., Fisher, B.L. \& Ward, P.S. (2006) Evaluating alternative hypotheses for the early evolution and diversification of ants. Proceedings of the National Academy of Sciences of the United States of America, 103, 18172-18177.

Brown, W.L. (1973) A comparison of the Hylean and Congo-West African rain forest ant faunas. In: B. J. Meggers, E. S. Ayensu \& W. D. Duckworth (Eds), Tropical forest ecosystems in Africa and South America: a comparative review. 
Smithsonian Institution Press, Washington, DC, pp. 161-185.

Buren, W.F. (1959) A review of the species of Crematogaster sensu stricto, in North America (Hymenoptera: Formicidae), part I. Journal of the New York Entomological Society, 66, 119-134.

Dalla Torre, K.W.v. (1893) Catalogus Hymenopterorum hucusque descriptorum systematicus et synonymicus. Engelmann, W., Leipzig, 289 pp.

Emery, C. (1922) Hymenoptera, fam. Formicidae, subfam. Myrmicinae. In: Genera Insectorum 174B, pp. 95-206.

Feldhaar, H., Fiala, B., Gadau, J., Mohamed, M. \& Maschwitz, U. (2003) Molecular phylogeny of Crematogaster subgenus Decacrema ants (Hymenoptera: Formicidae) and the colonization of Macaranga (Euphorbiaceae) trees. Molecular Phylogenetics and Evolution, 27, 441-452.

Feldhaar, H., Foitzik, S. \& Heinze, J. (2008) Lifelong hybridization to the wrong partner: hybridization in ants. Philosophical Transactions of the Royal Society of London B Biological Sciences, 363, 2891-2899.

Feldhaar, H., Gadau, J. \& Fiala, B. (2010) Speciation in obligately plant-associated Crematogaster ants: Host-distribution rather than adaption towards specific hosts drives the process. In: M. Glaubrecht (Ed), Evolution in Action Springer, Berlin, Germany, pp. 193-213.

Fiala, B., Jakob, A., Maschwitz, U. \& Linsenmair, K.E. (1999) Diversity, evolutionary specialization and geographic distribution of a mutualistic ant-plant complex: Macaranga and Crematogaster in South East Asia. Biological Journal of the Linnean Society, 66, 305-331.

Fisher, B.L. (2002) Ant diversity patterns along an elevational gradient in the Réserve Spéciale de Manongarivo, Madagascar. Boissiera, 59, 311-328.

Fisher, B.L. (2003) Formicidae, ants. In: S. M. Goodman \& J. P. Benstead (Eds), The Natural History of Madagascar. The University of Chicago Press, Chicago, pp. 811-819.

Fisher, B.L. (2005) A model for a global inventory of ants: A case study in Madagascar. Proceedings of the California Academy of Sciences, 56, 86-97.

Fisher, B.L. \& Penny, N.D. (2008) Les arthropodes. In: S. M. Goodman (Ed), Paysage naturels et biodiversité de Madagascar. Museum National de Histoire Naturelle, Paris, pp. 183-212.

Forel, A. (1887) Fourmis récoltées à Madagascar par le Dr. Conrad Keller. Mitteilungen der Schweizer Entomologischen Gesellschaft, 7, 381-389.

Forel, A. (1891) Les Formicides. [part]. In: A. Grandidier (Ed), Histoire physique, naturelle, et politique de Madagascar. Volume XX. Histoire naturelle des Hyménoptèrés. Deuxième partie (28e fascicule). Hachette et Cie, Paris, p. 1-280.

Forel, A. (1892) Nouvelles espèces de formicides de Madagascar (Récoltées par M. Sikora). Annales de la Société Entomologique de Belgique, 36, 516-535.

Forel, A. (1901) Variétés myrmécologiques. Annales de la Société Entomologique de Belgique, 45, 334-382.

Forel, A. (1910a) Glanures myrmécologiques. Annales de la Société Entomologique de Belgique, 54, 6-32.

Forel, A. (1910b) Zoologische und anthropologische Ergebnisse einer Forschungsreise im westlichen und zentralen Südafrika ausgeführt in den Jahren 1903-1905 von Dr. Leonhard Schultze. Vierter Band. Systematik und Tiergeographie. D) Formicidae. Denkschriften der Medizinsch-Naturwissenschaftlichen Gesellschaft Jena, 16, 130 .

Gautier, L. \& Goodman, S.M. (2002) Description de la Réserve Spéciale de Manongarivo, Madagascar. Boissiera, 59, $21-40$.

Gautier, L. \& Goodman, S.M. (2003) Introduction to the Flora of Madagascar. In: S. M. Goodman \& J. P. Benstead (Eds), The Natural History of Madagascar. Chicago University Press, Chicago, pp. 229-250.

Goodman, S.M. \& Benstead, J.P. (2005) Updated estimates of biotic diversity and endemism for Madagascar. Oryx, 39, 73-77.

Harris, R.A. (1979) A glossary of surface sculpturing. Occasional papers in Entomology, 28, 1-31.

Hölldobler, B. \& Wilson, E.O. (1990) The ants. Cambridge, Massachussetts: Harvard University Press, 732 pp.

Hosoishi, S. \& Ogata, K. (2009) A taxonomic revision of the Asian endemic subgenus Physocrema of the genus Crematogaster (Hymenoptera: Formicidae). Zootaxa, 2062, 15-36.

Hosoishi, S. \& Ogata, K. (2010) On the identity of Crematogaster schimmeri Forel, 1912 and the distribution of subgenus Decacrema in Asia (Hymenoptera: Formicidae). Asian Myrmecology, 3, 1-3.

Hosoishi, S., Yamane, S. \& Ogata, K. (2010) Subterranean species of the ant genus Crematogaster in Asia (Hymenoptera: Formicidae). Entomological Science, 13, 345-350.

International Commission on Zoological Nomenclature (1999) International Code of Zoological Nomenclature, amended $4^{\text {th }}$ edition, online version. Available from http://www.nhm.ac.uk/hosted-sites/iczn/code/ (accessed 24 September 2010).

Korczynska, J., Gajewska, M., Pilot, M., Czechowski, W. \& Radchenko, A. (2010) Genetic polymorphism in "mixed" colonies of wood ants (Hymenoptera: Formicidae) in southern Finland and its possible origin. European Journal of Entomology, 107, 157-167.

Kremen, C., Cameron, A., Moilanen, A., Phillips, S.J., Thomas, C.D., Beentje, H., et al. (2008) Aligning conservation priorities across taxa in Madagascar with high-resolution planning tools. Science (Washington D C), 320, $222-226$. 
Longino, J.T. (2003) The Crematogaster (Hymenoptera, Formicidae, Myrmicinae) of Costa Rica. Zootaxa, 151, 1-150.

Lund, P.W. (1831) Lettre sur les habitudes de quelques fourmis du Brésil, adressée a M. Audouin. Annales des Sciences Naturelles, 23, 113-138.

Mann, D.W. (1919) The ants of the British Solomon islands. Bulletin of the Museum of Harvard, 63, $273-301$.

Marlier, J.F., Quinet, Y. \& de Biseau, J.C. (2004) Defensive behaviour and biological activities of the abdominal secretion in the ant Crematogaster scutellaris (Hymenoptera: Myrmicinae). Behavioural Processes, 67, 427-440.

National Geospatial-Intelligence Agency (2010) GEOnet Names Server. Available from http://earth-info.nga.mil/gns/ html/index.html (accessed 24 September 2010).

Myers, N., Mittermeier, R.A., Mittermeier, C.G., da Fonseca, G.A.B. \& Kent, J. (2000) Biodiversity hotspots for conservation priorities. Nature (London), 403, 853-858.

Quek, S.-P., Davies, S.J., Itino, T. \& Pierce, N.E. (2004) Codiversification in an ant-plant mutualism: stem texture and the evolution of host use in Crematogaster (Formicidae: Myrmicinae) inhabitants of Macaranga (Euphorbiaceae). Evolution, 58, 554-570.

Santschi, F. (1918) Sous-genres et synonymies de Cremastogaster. Bulletin de la Société Entomologique de France, 1918, 182-185.

Santschi, F. (1928) Nouvelles fourmis de Chine et du Turkestan Russe. Bulletin et Annales de la Société Entomologique de Belgique, 68, 31-46.

Schatz, G. \& Lescot, M. (2003) Gazetteer to Malagasy Botanical Collecting Localities. Available from http:// www.mobot.org/mobot/research/madagascar/gazetteer/ (accessed 24 September 2010).

Smith, D.R. (1979) Superfamily Formicoidea. In: Krombein, K. V., Hurd, P. D., Smith, D. R., Burks, B. D. (Eds.) Catalog of Hymenoptera in America north of Mexico. Vol. 2. Apocrita (Aculeata). Smithsonian Institution Press, Washington, D.C., pp. 1323-1467.

Taylor, R. (1989) The nomenclature and distribution of some Australian ants of the genus Polyrhachis Fr. Smith (Hymenoptera: Formicidae: Formicinae). Journal of the Australian Entomological Society, 28, 23-27.

Taylor, R. (1991) Nomenclature and distribution of some Australasian ants of the Myrmicinae (Hymenoptera: Formicidae). Memoirs of the Queensland Museum, 30, 599-614.

United States Board on Geographical Names (1989) Gazetteer of Madagascar (2nd ed.). Defense Mapping Agency, Washington, D.C., 826 pp.

Vences, M., Wollenberg, K.C., Vieites, D.R. \& Lees, D.C. (2009) Madagascar as a model region of species diversification. Trends in Ecology \& Evolution, 24, 456-465.

Viette, P. (1991) Principales localités où des Insectes ont été recueillis à Madagascar. Faune de Madagascar, Supplement 2, Viette, P, $88 \mathrm{pp}$.

Ward, P.S. (2001) Taxonomy, phylogeny and biogeography of the ant genus Tetraponera (Hymenoptera:Formicidae) in the Oriental and Australian regions. Invertebrate Taxonomy, 15, 589-665.

Ward, P.S. (2007) Phylogeny, classification, and species-level taxonomy of ants (Hymenoptera: Formicidae). Zootaxa, 1668, 549-563.

Ward, P.S. (2010) Taxonomy, Phylogenetics and Evolution. In: L. Lach, C. L. Parr \& K. L. Abott (Eds), Ant Ecology. Oxford University Press, Oxford, $402 \mathrm{pp}$.

Ward, P.S. \& Downie, D.A. (2005) The ant subfamily Pseudomyrmecinae (Hymenoptera : Formicidae): phylogeny and evolution of big-eyed arboreal ants. Systematic Entomology, 30, 310-335.

Weissflog, A. (2001) Freinestbau von Ameisen (Hymenoptera: Formicidae) in der Kronenregion feuchttropischer Wälder Südostasiens: Bestandsaufnahme und Phänologie, Ethoökologie und funktionelle Analyse des Nestbaus. Ph.D. Thesis, University of Frankfurt, Germany. Available from http://publikationen.ub.uni-frankfurt.de/volltexte/ 2006/3287/ (accessed 29 September 2010).

Wheeler, W.M. (1911) A list of the type species of the genera and subgenera of Formicidae. Annals of the New York Academy of Sciences, 21, 157-175.

Wheeler, W.M. (1922a) Ants of the American Museum Congo expedition. A contribution to the myrmecology of Africa. VII. A synonymic list of the ants of the Malagasy region. Bulletin of the American Museum of Natural History, 45, 631-710.

Wheeler, W.M. (1922b) Ants of the American Museum Congo expedition. A contribution to the myrmecology of Africa. IX. Keys to the genera and subgenera of ants. Bulletin of the American Museum of Natural History, 45, $1005-1055$.

Wheeler, W.M. (1927) The physiognomy of insects. Quarterly Review of Biology, 2, 1-36.

Wheeler, W.M. (1936) A singular Crematogaster from Guatemala. Psyche (Camb.), 43, 40-48.

Yoder, A.D. \& Nowak, M.D. (2006) Has vicariance or dispersal been the predominant biogeographic force in Madagascar? Only time will tell. Annual Reviews in Ecology, Evolution and Systematics, 37, 405-431. 